\title{
Opportunities in vanadium-based strongly correlated electron systems
}

\author{
Matthew Brahlek, Lei Zhang, Jason Lapano, Hai-Tian Zhang, and Roman Engel-Herbert, Department of Materials Science and \\ Engineering, Pennsylvania State University, University Park, Pennsylvania 16801, USA \\ Nikhil Shukla, and Suman Datta, Department of Electrical Engineering, University of Notre Dame, Notre Dame, Indiana 46556, USA \\ Hanjong Paik, and Darrell G. Schlom, Department of Material Science And Engineering, Cornell University, Ithaca, New York 14853, USA \\ Address all correspondence to Roman Engel-Herbert at rue2@psu.edu
}

(Received 12 October 2016; accepted 12 January 2017)

\begin{abstract}
The diverse and fascinating properties of transition metal oxides stem from the strongly correlated electronic degrees of freedom; the scientific challenge and range of possible applications of these materials have caused fascination among physicists and materials scientists, thus capturing research efforts for nearly a century. Here, we focus on the binary $\mathrm{V}_{x} \mathrm{O}_{y}$ and the ternary perovskite $\mathrm{AVO}_{3}$ and review the key aspects from the underlying physical framework and their basic properties, recent strides made in thin-film synthesis, to recent efforts to implement vanadium-based oxides for practical applications that augment existing technologies, which surpass limitations of conventional materials.
\end{abstract}

\section{Introduction}

Living in the information age we are surrounded by technology that enables us to solve problems on a daily basis, which were thought to be intangible just a couple of decades ago. High-performance computation, ubiquitous communication as well as instant storage of large sets of data have allowed information technology to transform virtually all aspects of our lives and has changed society in an irrevocable way: computer-aided design strategies to develop new products, realistic simulations to predict and verify their functionalities, control of robotic manufacturing processes, and the role of computers to orchestrate complex processes, such as managing supply chains in industry or large-scale science experiments. Furthermore, new social media have emerged and videoconferencing platforms enable fast, high quality, personal interaction in affordable ways. Similarly, cloud computing allows sharing large data sets and provides concurrent access from all over the world in real time. All these examples attest to the ways in which information technology has revolutionized how we construct, build, communicate, and understand our world.

Without any doubt, semiconductor materials in general, and silicon in particular, constitute the material basis for all these opportunities. In semiconductors, two key functionalities are ideally met-(1) modulating the flow of charge carriers by an applied electric field thereby enabling manipulation, amplification, and storage of electrical signals; and (2) transducing electrical signals into optical signals and vice versa at very fast time scales. Improved synthesis and fabrication technologies of these materials, the development of nanoscale metrology tools, and consequent exploitation of computers to simulate devices and understanding materials from first-principles have formed a self-feeding cycle of innovation and technology advancements. This enables the continuous improvement of device performance, faster operation, and increased level of integration density at lower costs that is witnessed today. But, how long will this process continue and what will come next?

Gordon Moore's prediction ${ }^{[1]}$ to double the number of transistors every 2 years has held for the past four decades with a near million-fold increase in transistor density. In the mid-2000s, single-thread microprocessor clock speed stagnated to limit heat dissipation, which marked the end of classical scaling and the introduction of power-constraint scaling. ${ }^{[2]}$ Multi-core architectures have been used to continue enhancing computing performance per chip keeping the projected pace. At present, however, continued scaling has slowed down to a 3 -year turnaround. It is anticipated that the $10 \mathrm{~nm}$ technology node will be introduced in 2017, while the $7 \mathrm{~nm}$ node is projected for 2020, owed to the ever increased complexity of the chip manufacturing process. ${ }^{[3]}$ The slowing cadence of Moore's Law signals that profitability of advancements are becoming smaller, thus favoring a strategy to look for new material opportunities to augment the current hardware and expand beyond the current complimentary metal-oxide-semiconductor (CMOS) technology. With silicon being the material of the 20th century, emerging electronic materials of the 21 st century will add novel functionalities not feasible in conventional semiconductors, thereby permitting higher throughput and more energy-efficient hardware solutions for computation, communication, and data storage.

Transition metal oxides (TMOs) represent such a class of materials. While the rationale for honing in on $\mathrm{Si}$ and other semiconductors was their electronic response to external 
electric field, robustness, ease of surface passivation, and excellent electronic transport properties at room temperature, the conduction bands in TMOs are derived from $d$-orbitals stemming from the transition metal element, which provide additional functional complexity if partially filled. As graphically depicted in Fig. 1, electrons in a solid have three fundamental properties: charge, spin, and orbital degrees of freedom (DOF). In conventional semiconductors, the completely filled bands quench the spin and orbital DOF, which in turn do not play any role, leaving charge modulation via field effect as the primary control mechanism. In contrast, in TMOs the spin and orbital DOF are active and coupled, which can give rise to frustration resulting in a manifold of competing ground states with different order phenomena, and, therefore, coupled phase transitions. For example, the proximity to the fluctuating anti-ferromagnetic phase is thought to be key for the stabilization of the high-temperature (high- $T_{\mathrm{C}}$ ) superconducting state in the complex cuprate superconductors. ${ }^{[4]}$ Furthermore, crosscoupling of magnetic and orbital order can drive fielddependent metal-insulator transitions (MITs) that give rise to colossal magnetoresistive effects in manganites. ${ }^{[5]}$ TMOs have widely been the focus of fundamental research, with extensive emphasis placed on peculiar properties of the manganites $\left(A, A^{\prime}\right) \mathrm{MnO}_{3}$ where $A$ and $A^{\prime}$ are, respectively, rare-earth and alkali-earth cations, nickelates $\left(A, A^{\prime}\right) \mathrm{NiO}_{3}$, and the

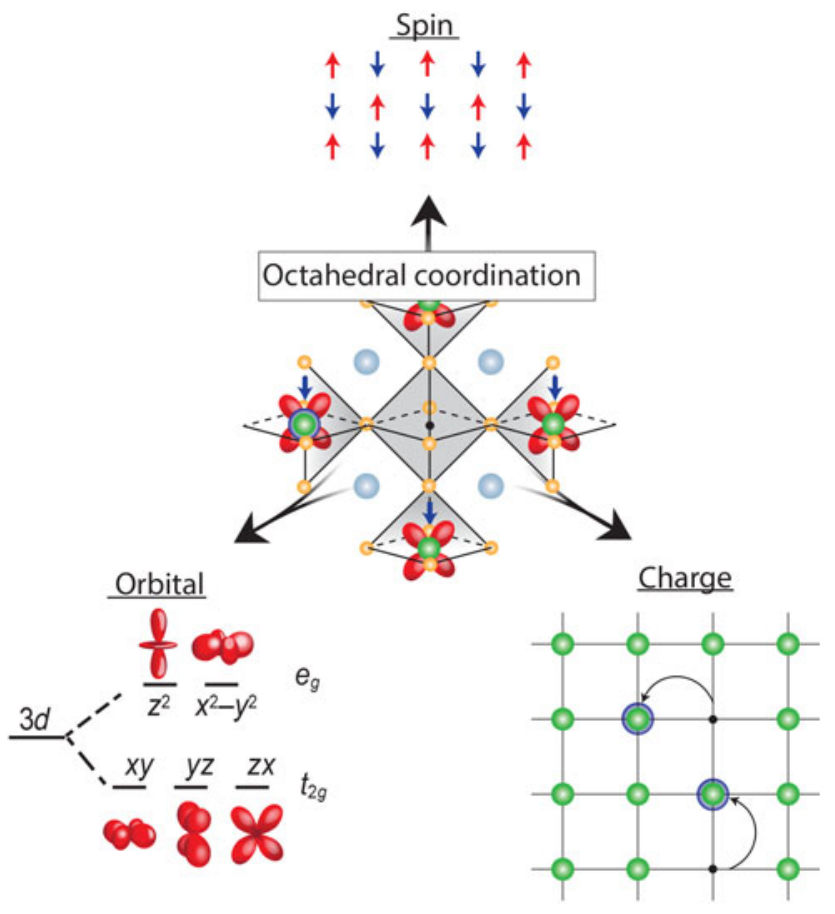

Figure 1. Octahedral coordination of the vanadium cation as the fundamental building block of properties in all vanadium oxide phases. The strong electron correlation effects couple the three electron DOF, spin, orbital, and charge; which can result in frustration and competing ground states with different long-range order, electronic and optical properties that give rise to the wide range of properties observed in vanadium oxide compounds. chemically more complicated layered cuprates (for example, $\mathrm{HgBa}_{2} \mathrm{Ca}_{2} \mathrm{Cu}_{3} \mathrm{O}_{8}$, with the highest $T_{\mathrm{C}}$ of $\left.134 \mathrm{~K}^{[6]}\right) .{ }^{[7-9]}$

In contrast to these TMOs containing middle- and late-transition-metal elements, vanadium-based (V-based) oxides have been far less studied. The close proximity of electronic phases with disparate electronic properties observed in $\mathrm{V}$-based oxides makes them attractive as electronic phase change materials (PCMs). Unlike in the case of semiconductors, which have been perfected over decades to the highest level of maturity, we are only at the beginning to learn how to stabilize and control these electronic phases and how to put them to use in practical devices. Here, we review recent advances and project future opportunities that V-based TMO materials possess. We focus on binary and ternary V-based oxides, which show a wide range of ground states ranging from metals to insulators, coupled charge-, spin-, and orbital-ordered phases, to superconductors (see Refs. 9-13 and reference therein). The outline is as follows: In the section "Strong electron correlations in V-based oxides", the physical concepts underpinning the understanding of electronic properties in V-based oxides are outlined and how these drastically differ from semiconductor materials. In the section "MIT transition in binary vanadium compounds: $\mathrm{VO}_{2}$ and $\mathrm{V}_{2} \mathrm{O}_{3}$ " the challenges to synthesize specific binary vanadium oxides $\left(\mathrm{V}_{x} \mathrm{O}_{y}\right)$ is discussed and how better control over the deposition has recently enabled progress in the quality of ultrathin $\mathrm{VO}_{2}$ films. Three specific examples are reviewed how the electronic $\mathrm{PCM} \mathrm{VO}_{2}$ has recently been applied to create practical electronic devices with competitive functionality (1) by augmenting metal-oxide-semiconductor field effect transistors (MOSFET), enabling a positive feedback in the turn-on characteristic to surpass the Boltzmann limit of $60 \mathrm{mV} /$ decade (section "Augmenting field effect transistors (FETs) with $\mathrm{VO}_{2}$ "); (2) to serve as a scalable hardware platform to enable non-Boolean computing schemes via synchronization behavior through capacitively coupled charge oscillator devices stabilized in their phase coexistence regime (Section "Non-Boolean computing using charge coupled $\mathrm{VO}_{2}$ oscillators"); and (3) as an electronic switch, uniquely balancing the high operation frequency of microelectromechanical systems (MEMS) switches with the reliability and fast switching characteristics of a transistor-based solid-state radio frequency (RF) switch (section "VO ${ }_{2}$ as RF switch"). In the section "Ternary oxide vanadates", the discussion is expanded toward ternary vanadium oxide phases. Starting by exemplifying the strong electron correlation effects in $\mathrm{SrVO}_{3}$ and $\mathrm{CaVO}_{3}$ in the section "Ternary oxide vanadates". A the challenges in growing thin films and how recent advances have made strides to simplify the growth procedure while improving material quality is reviewed in Section (Thin-film growth of $\mathrm{AVO}_{3}$ perovskites). As discussed in the section "A route to enhanced correlation effects: thickness-driven MIT" thin-film epitaxy can be used as a route to enhance correlation effects through dimensional confinement to drive the strongly correlated metal $\mathrm{SrVO}_{3}$ into an insulating phase. Then, as a direct application of the 
electron-correlation physics, section "Correlated metals as TCs" discusses how the specific band structure of vanadates and pronounced electron interaction effects inherent to the correlated metals $\mathrm{SrVO}_{3}$ and $\mathrm{CaVO}_{3}$ offer a novel design strategy to strike a better balance in the mutually exclusive properties of optical transparency and electrical conductivity, thus constituting a new paradigm in field of transparent conducting thin films. In section "The Mott-insulating phase in rare-earth vanadates", opportunities in the rare-earth perovskites $\left(R \mathrm{VO}_{3}\right)$ centered on the strongly spin-orbital coupled, Mott-insulating ground state for photovoltaic applications are reviewed. We finally close with an outlook about future vanadium oxide materials and their prospects for their application into the next generation of optoelectronic devices.

\section{Strong electron correlations in V-based oxides}

The atomic scale interactions that determine the macroscale electronic properties of a solid are complex. Deriving a solution for the many-body wave function of allowed electronic states that considers the Coulomb interaction between electrons and ions constituting the crystalline lattice, but also the repulsive interaction among all electrons, is impossible. For a large number of materials satisfactory predictions of electronic properties, however, can be obtained by employing the Hartree-Fock formalism (see, e.g., Ref. 14) where all many-body interactions except the electron-electron interaction are incorporated. The ground state is then described by a wave function of single, non-interacting quasi-particles (herein, unless otherwise stated electrons will refer to quasi-particles) that are considered nearly free and independent, i.e., not correlated, and can be computed self-consistently. The interactions with the periodic arrangement of ions forming the crystal are entirely accrued into a rescaled quasi-particle mass $m_{b}{ }^{*}$ - the electron effective mass in the respective band $b$. For semiconductors, where the conduction and valence band are derived from $s$ - and $p$-like orbitals, this nearly-free-electron picture provides an accurate description. This is made possible by the large kinetic energy of the carriers $\hbar^{2} k^{2} /\left(2 m_{b} *\right)$, with $\hbar$ being the reduced Planck constant and $k$ the electron wavevector that is of the order of the inverse lattice parameter, relative to the repulsive Coulomb interaction energy among the electrons. In conventional semiconductors, the kinetic energy is of the order of $10-20 \mathrm{eV}^{[15]}$ due to small electron effective mass that is typically one-tenth that of an free-electron mass. The electron-electron interaction, the potential energy of two electrons getting into very close proximity, i.e., being at the same lattice site, is much smaller, $\sim 1-2 \mathrm{eV}^{[16]}$ and can therefore be neglected. In this case, electrons barely affect each other, they are nearly free and their motion is not correlated.

In contrast, for many TMOs, including vanadium oxide phases, the approximation of the many-particle problem using the picture of nearly free electrons is found to not adequately describe real materials. Here, the kinetic energy and the Coulomb potential energy that arises from electron-electron interactions are of similar magnitude. The conduction and valence bands in conventional semiconductors stem from $s$ and $p$-hybridized orbitals, which have significant overlap giving rise to a large band width, sizeable band dispersion, and, thus a small effective mass and large kinetic energies; in contrast, the localized $3 d$ orbitals in TMOs form the partially occupied conduction band. The far more spatially localized and more directional shape of $3 d$ orbitals cause a much smaller orbital overlap, and thus smaller band width $W$ and larger effective masses that are similar to or even larger than the mass of the free electron in vacuum. A one order of magnitude increase in the free-electron mass directly reduces the electron's kinetic energy in the TMO $d$-bands making it comparable with the Coulomb repulsion, thus rendering the simple non-interacting picture invalid.

To illustrate how electron correlation effects modify the underlying physics, Fig. 2 schematically shows a comparison of the optical conductivity $\sigma(E)$ [Fig. 2(a)], the band structure with conduction band width $W$ and band gap $E_{g}$, i.e., the energy momentum relation $E(k)$ of electrons, along with the associated density of states, DOS [Fig. 2(b)]. In addition, the spectral function of the electrons $A(k, \omega)$ is shown as well [Fig. 2(c)], which represents the distribution of an ensemble of electrons having momentum $k$ and energy $E=\hbar \omega$. We first consider the optical conductivity. In general, photoexcitation causes a complex response of the many-body ground state; however, for uncorrelated materials, i.e., materials that have a negligible electronelectron interaction expressed by a vanishing Coulomb potential $(U=0)$, these excitations can be understood using the concept of non-interacting quasi-particles. For band insulators, the lowest possible excitation is to promote single electrons from filled states in the valence band (blue), to unoccupied states in the conduction band (white). The interband transition appears as the red shaded peak in $\sigma(E)$. For the case of a conventional metal with uncorrelated electrons, where the Fermi level $E_{\mathrm{F}}$ intersects the conduction band, lower-energy excitations are possible, because transitions from occupied to unoccupied states are possible within the same band. These intraband transitions with small excitation energies appear as the low-energy Drude peak (shaded yellow) in the optical conductivity. For these materials with negligible correlation effects, represented by a vanishing electron-electron interaction potential $U$, the respective spectral function shown in Fig. 2(c) is simply $A(k, \omega) \sim \delta[\omega-E(k)]$. It implies that electrons are described by orthogonal single-particle eigenstates with infinite lifetimes, and can be distinguished by wavevector $k$, the main quantum number, which is associated with a specific energy $E$. This single-valued relation, the dispersion relation $E=\hbar^{2} k^{2}$ / $\left(2 m_{b}{ }^{*}\right)$, is a direct result that electrons only interact with the periodic lattice potential.

The addition of an electron-electron interaction breaks the ideal periodicity of the underlying lattice, thus requiring a modified framework to properly account for experimental observations. As electron correlation becomes sizeable $(U>0)$ additional features in the optical conductivity appear. As 
No correlation: $U=0$

(a)

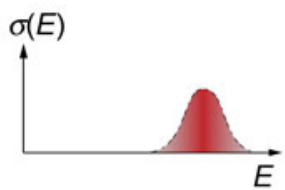

Band insulator

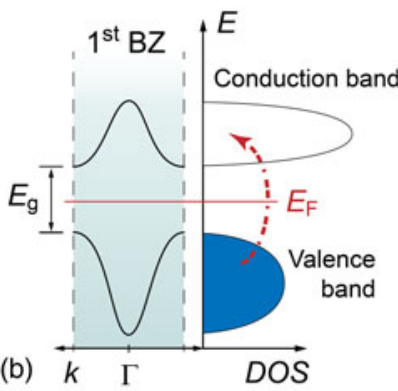

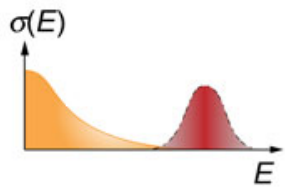

Conventional metal

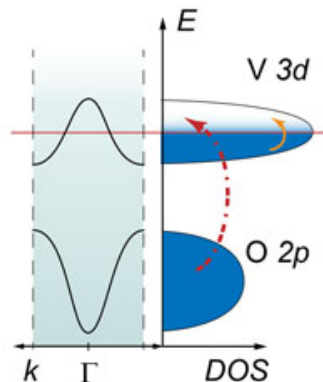

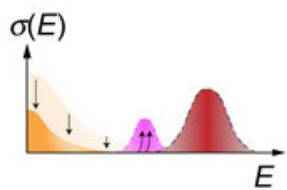

Correlated metal: $U<W$
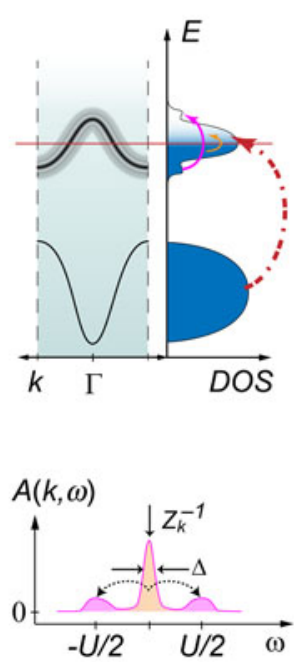

(c)

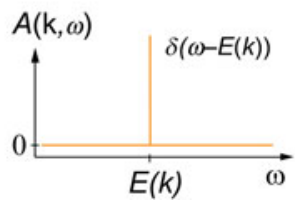

Mott insulator: $U>W$


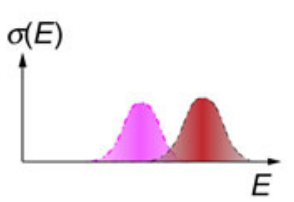

Strong correlation: $U>0$

0



\begin{abstract}
Figure 2. Comparison of various properties for systems without electron correlation effects (left) and strong electron correlated (right): (a) optical conductivity $\sigma$ $(E)$, (b) the band structure $\varepsilon(k)$ and density of states DOS, (c) the spectral function $A(k, \omega)$. Dashed and solid curves in (b) represent inter- and intraband transitions, giving rise to a peak (red) and the Drude tail (yellow) in the optical conductivity. Solid and dashed arrows in (c) illustrate the changes in the spectral function $A(k, \omega)$ with increasing correlation strength, causing reduction and widening $\left(\Delta \sim Z_{k}^{-1}\right)$ of the coherent peak at $\omega=0$ and a spectral weight transfer to the incoherent side peaks, respectively.
\end{abstract}

shown on the right side of Fig. 2(a), the magnitude of the Drude peak at low energy decreases and a new peak, shaded pink, at higher energy appears for $U<W$. This change is reflected in the $D O S$ as well where lobes emerge at the conduction band edges and the magnitude at the Fermi energy is diminished. Electrons are now correlated, which implies that their energies now also depend on the spatial arrangement of adjacent electrons in the near vicinity. Even being in the same state, represented by wavevector $k$, they can be in different local configurations and thus at different energies, which is captured by a more complex spectral function $A(k, \omega)$ shown in Fig. 2(c). Note that the magnitude of the coherent peak in the spectral function of the electron, representing delocalized, free moving quasi-particles, is renormalized and broadened by the factor $Z_{k}$. Additionally, incoherent side peaks emerge at $E= \pm U / 2$ relative to the coherent peak. Electrons in correlated systems can have a range of energies with a span of the Columbic interaction energy $U$, because despite a very well-determined momentum defining the kinetic energy it can be in different local electron arrangements, ranging from virtually no other electrons in its spatial vicinity energy, to experiencing the maximum Coulomb effect $U$ from the electron-election interaction. The local nature breaks the periodic potential that makes the band structure well defined, and the dispersion relation becomes smeared, schematically illustrated in Fig. 2(b).

The correlation effects can be captured by renormalizing the band mass $m_{\mathrm{b}} *$ by $m^{*}=m_{\mathrm{b}} * / Z_{k}$, which reduces the kinetic energy, the band width $W$ and thus the itinerant character of electrons compared with results obtained in the Hartree-Fock approximation. The band dispersion is not a single-valued function anymore, where for each wavevector $k$ only a very specific energy value is found. Instead it can be considered as convolution of the spectral function with the band structure to account for the distribution of different local electron configurations. This is in dramatic difference to conventional materials with negligible electron-electron interaction. In the presence of sizeable electron correlation, the band structure also depends on the spatial arrangement of the electrons. Therefore band structure can be potentially altered in response to changes of the spatial arrangement of electrons.

Further increasing electron correlation strength, represented by $U$, electrons are becoming less itinerant and more become localized, which is captured by a further spectral weight transfer from the coherent to incoherent part in $A(k, \omega)$, a reduced life 
time and magnitude of the itinerant quasi-particle $\left(0<Z_{k}<1\right)$. In the extreme limit of electron correlation, $U>W$ and $Z_{k}=0$ and the coherent peak in $A(k, \omega)$ vanishes and hence the quasiparticle effective mass of itinerant electrons diverges and their lifetime approaches zero: they no longer exist. Only the incoherent peaks remain forming the lower and upper Hubbard band separated by the Mott gap $E_{\mathrm{M}}$. While the lower, fully occupied Hubbard band represents the singly occupied crystal site, the upper, unoccupied Hubbard band represents the doubly occupied site, and, therefore the energy separation is proportional to the Coulomb energy $U$ of interacting electrons.

This correlation-induced transition from a metallic phase to an insulating ground state, as proposed by N. F. Mott, is the underlying physics of the early transition metal oxides. ${ }^{[17,18]}$ While TMOs with partially filled $d$-bands are expected to be metallic from simple band structure arguments, electrons are being localized due to the strong electron-electron interaction and hence do not contribute to conduction making the material electrically insulating. The Mott transition is directly tied to the local bonding environment by the electron interaction strength $U$ and orbital overlap determining the band width $W$. Therefore, the bonding environment is key to understand and control the ground state. While the valence state of vanadium can range from $2^{+}-5^{+}$the associated ionic radii only allow for sixfold coordination when bonded to oxygen according to Pauling's rules $^{[19]}$. In case of vanadium, it therefore takes the shape of an oxygen octahedron with $\mathrm{V}$ located inside, as shown in the center of Fig. 1. This forms the fundamental building block of virtually all V-based oxide materials, mediating the complex coupling between the competing charge, spin, and orbital DOF.

\section{MIT in binary vanadium compounds: $\mathrm{VO}_{2}$ and $\mathrm{V}_{\mathbf{2}} \mathrm{O}_{3}$}

Among the materials exhibiting MITs, perhaps the most intensely studied compounds are the binary oxides of vanadium. ${ }^{[10,20]}$ In particular, $\mathrm{V}_{2} \mathrm{O}_{3}$, the trivalent oxide of vanadium, exhibits a well-studied MIT from a high-temperature paramagnetic metallic (PM) to a low-temperature AFI (antiferromagnetic insulating) state at $154 \mathrm{~K}$, along with a change in electrical conductivity by eight orders of magnitude. ${ }^{[21]}$ Similarly an MIT occurs in the tetravalent compound $\mathrm{VO}_{2}$ at $340 \mathrm{~K}$, but in contrast to $\mathrm{V}_{2} \mathrm{O}_{3}$ it transitions from a hightemperature PM state to a low-temperature, PI (paramagnetic insulator state), exhibiting five orders of magnitude change in conductivity. ${ }^{[22]}$ Symmetry-lowering structural distortions accompany the MITs of $\mathrm{V}_{2} \mathrm{O}_{3}$ and $\mathrm{VO}_{2}$ making them a firstorder transition. Despite extensive investigations since the first observations of this phenomena dating back more than half a century, the exact mechanisms and intricate interplays of the different DOF driving the MITs are still controversially discussed, while structure and electronic properties have been characterized in detail are comprehensively understood. ${ }^{[10,23,24]}$

$\mathrm{V}_{2} \mathrm{O}_{3}$ in the high-temperature metallic state crystallizes into the corundum structure (space group $R \overline{3}$ c); see Fig. 3(a).
Vanadium is displaced out of the center of the oxygen octahedra away from the nearest neighboring vanadium. ${ }^{[2]}$ The crystal field splits the V $3 d$ orbitals into the high-energy, doubly degenerate $e_{g}^{\sigma}$ states and the low-energy, triply degenerate $t_{2 g}$ states, the latter occupied by both valence electrons with a total spin $S=1$ per $\mathrm{V}^{3+}$ lattice site. ${ }^{[24]}$ The degeneracy of the partially filled $t_{2 g}$ states is lifted due to a trigonal distortion of the octahedron, which then split into a high-energy, nondegenerate $a_{1 g}$ state and two low-energy, doubly degenerate $e_{g}^{\pi}$ states. While the derived $a_{1 g}$ band would be nominally empty, the lower lying $e_{g}^{\pi}$ band would be half-filled; however, due to hybridization of both states a significant band overlap results allowing for metallic conduction. ${ }^{[23]}$ Upon cooling through the transition temperature, $\mathrm{V}_{2} \mathrm{O}_{3}$ undergoes a structural transformation to the monoclinic $(I 2 / a)$ phase; see Fig. 3(b). ${ }^{[25]}$ The $\mathrm{V}$ is displaced within the oxygen octahedra such that it forms $\mathrm{V}-\mathrm{V}$ pairs through edge-sharing oxygen bonds. This dimerization is accompanied with a volume decreases by $1.3 \%$, the $c / a$ ratio increases by $1.4 \%$, and the $e_{g}^{\pi}$ band is pushed below the $a_{1 g}$ band by $\sim 0.5 \mathrm{eV} \cdot{ }^{[24]}$ The distortion increases the exchange interaction between nearest-neighbor vanadium atoms and leads to the coincident antiferromagnetic ordering of the $t_{2 g}$ orbitals; however, the exact occupation of electrons within these orbitals is still not fully understood. ${ }^{[24,26-29]}$

Despite the large change in resistivity arising from the formation of an energy gap separating the highest occupied from the lowest unoccupied state, the MIT in $\mathrm{V}_{2} \mathrm{O}_{3}$ occurs at too low temperatures to be of practical relevance for applications. A transition temperature of $340 \mathrm{~K}$ makes $\mathrm{VO}_{2}$ more attractive for applications, similar to the less studied $\mathrm{V}_{3} \mathrm{O}_{5}$ phase also exhibiting an MIT above room temperature $\left(150{ }^{\circ} \mathrm{C}\right)$, albeit with a much smaller change in resistivity. ${ }^{[30,31]} \mathrm{VO}_{2}$ is paramagnetic in its high symmetry metallic state with rutile structure (space group $P 4_{2} / \mathrm{mmm}$ ), shown in Fig. 3(c), and undergoes an MIT to the monoclinic (M1) insulating phase. ${ }^{[20]}$ Similar to $\mathrm{V}_{2} \mathrm{O}_{3}$, in the high-temperature metallic phase, the $t_{2 g}$ orbitals are split into an $a_{1 g}$ and two $e_{g}^{\pi}$ states that overlap. ${ }^{[2]} \mathrm{As}_{\mathrm{VO}_{2}}$ is cooled through the MIT transition the symmetry is lowered via a monoclinic distortion that is associated with V-pair formation (dimerization); see Fig. 3 (d). The $a_{1 g}$ band is then further split into an upper and lower part while the $e_{g}^{\pi}$ band is shifted up, leading to the formation of a gap between the $e_{g}^{\pi}$ and the lower $a_{1 g}$ bands.

The transitions in both $\mathrm{VO}_{2}$ and $\mathrm{V}_{2} \mathrm{O}_{3}$ are sensitive to chemical and external pressure, epitaxial strain, as well as V- or O-defects. ${ }^{[10,32]}$ External pressure has been known to be an effective means to modulating the MIT transition in both $\mathrm{VO}_{2}$ and $\mathrm{V}_{2} \mathrm{O}_{3} \cdot{ }^{[22,33]}$ More recently, epitaxial strain has been used to either induce or suppress the metallic state. ${ }^{[34,35]}$ $\mathrm{V}_{2} \mathrm{O}_{3}$ grown on $\alpha-\mathrm{Al}_{2} \mathrm{O}_{3}(0001)(+3.9 \%$ biaxial strain) stabilized the metallic state to down to 2 from $170 \mathrm{~K}$, while the transition temperature was raised to $190 \mathrm{~K}$ for $\mathrm{V}_{2} \mathrm{O}_{3}$ samples grown on $\mathrm{LaTaO}_{3}(0001)\left(-4.0 \%\right.$ biaxial strain). ${ }^{[36]}$ Studies on $\mathrm{VO}_{2}$ have also shown compression of the $c$-axis for films grown 



Figure 3. Crystal structure (top) and energy-level diagram (bottom, adapted with permission from Ref. 24. Copyrighted by the American Physical Society) of $\mathrm{V}_{2} \mathrm{O}_{3}(\mathrm{a}, \mathrm{b})$ and $\mathrm{VO}_{2}(\mathrm{c}, \mathrm{d})$ in the high-temperature metal phase $(\mathrm{a}, \mathrm{c})$, and the low-temperature insulating phase $(\mathrm{b}, \mathrm{d})$. The red arrows indicate the atomic displacements across the structural transition.

on $\mathrm{TiO}_{2}(001)(+0.86 \%$ biaxial strain) shifting the transition temperature down to about $300 \mathrm{~K}$, making this system desirable for room-temperature-based devices. ${ }^{[35,37]}$

Coupling the transitions to an external electric field has long been sought-after as means to modulate the MIT. ${ }^{[38]}$ Such control, however, is difficult due to the large electric field needed to induce the metallic state, which may exceed the critical breakdown field of typical gate dielectrics commonly employed. Higher field strengths can be achieved with alternative techniques such as ferroelectric gates, ${ }^{[38]}$ polar interfaces, ${ }^{[39]}$ and more recently with ionic liquid double-layers, ${ }^{[40]}$ all of which can modulate sheet charge densities on the order of one electron per unit cell $\left(\sim 10^{15} / \mathrm{cm}^{2}\right)$. Although some reports show that a metallic phase can be induced in $\mathrm{VO}_{2}$ using ionic liquid gating technique, ${ }^{[41]}$ other experiments suggest that not the high electric fields causing the desired electrostatic doping, but rather electrochemical reactions affecting the film's stoichiometry might be responsible to induce the different phases. ${ }^{[2,43]}$ The following subsection "Thin-film growth of $\mathrm{VO}_{2}$ " reviews how binary oxides can be synthesized as epitaxial thin films and utilized in various applications, limiting ourselves to $\mathrm{VO}_{2}$.

\section{Thin-film growth of $\mathrm{VO}_{2}$}

Moving from the frontier of fundamental research in $\mathrm{VO}_{2}$ electronics toward their realization in commercial products, a method to deposit high-quality and ultrathin $\mathrm{VO}_{2}$ films on wafer scale is needed. For example, memory devices based on MITs have the advantage of compact node sizes down to a few $\mathrm{nm}$ and $\mathrm{VO}_{2}$ films must preserve their MIT properties during the scaling process, e.g., maintaining at least four orders of magnitude change in resistivity across the MIT. ${ }^{[44]}$ However, the MIT properties in $\mathrm{VO}_{2}$ thin films has long been recognized 
to be highly sensitive to deposition conditions including substrate temperature, background oxygen pressure, as well as deposition technique. This delicate nature of $\mathrm{VO}_{2}$ results from its complex phase diagram as shown in Fig. 4(a). The $\mathrm{VO}_{2}$ phase is not a line compound, and, thus, is intrinsically susceptible to point defect formation if growth conditions deviate
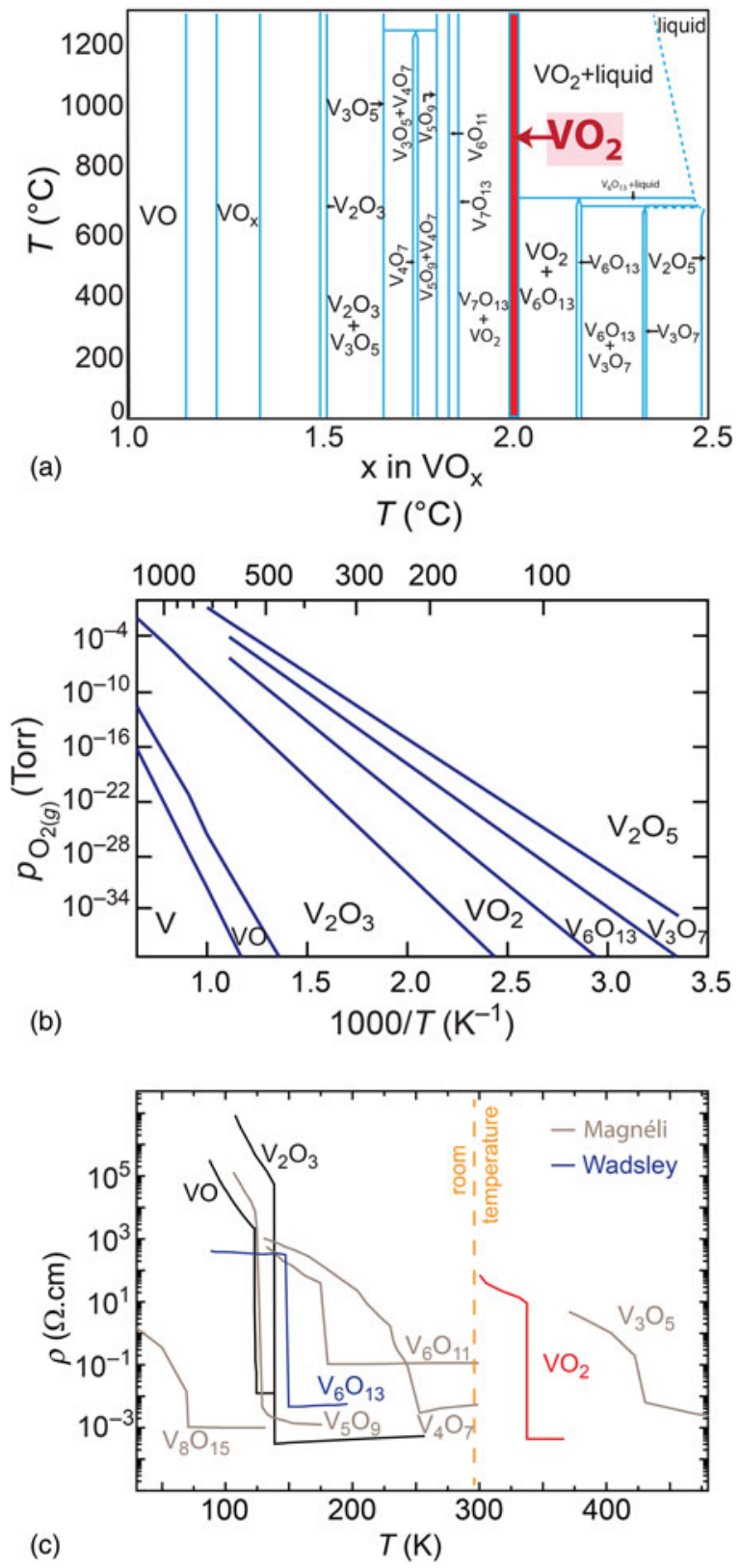

Figure 4. (a) Phase diagram of vanadium oxide, adapted permission from Ref. 47. Copyrighted by the American Physical Society. (b) The Ellingham diagram for vanadium oxide phases. ${ }^{[48,49]}$ (c) Resistivity versus temperature for various $\mathrm{V}-\mathrm{O}$ phases, with a MIT at different temperatures. While $\mathrm{V}_{2} \mathrm{O}_{3}$ exhibits the largest change in resistivity over eight orders of magnitude, but below room temperature, $\mathrm{VO}_{2}$ has the largest resistivity ratio above room temperature of about five orders of magnitude. ${ }^{[20,21,30,181-183]}$ slightly from the ideal ratio. Long-range order of these defects can occur forming distinctive phases. ${ }^{[45]}$ In the oxygen deficient case, $\mathrm{VO}_{2-\delta}$, the so-called Magnéli phases, $\mathrm{V}_{n} \mathrm{O}_{2 n-1}$, with $n=3-7$ form a homologous series with one oxygen layer missing after every $n$th vanadium (211) plane of the $\mathrm{VO}_{2}$ rutile structure. Similarly, the oxygen-rich $\mathrm{VO}_{2+\delta}$ Wadsley phases, $\mathrm{V}_{n} \mathrm{O}_{2 n+1}$ are known with $n=2,3$, and 6. ${ }^{[46,47]}$

The rich phase diagram, the different polymorphs and the ability to easily accommodate defects that can order and dramatically affect the MIT properties, makes the growth of a targeted vanadium oxide phase particularly challenging. ${ }^{[45]}$ Figure 4(b) shows the Ellingham diagram calculated from the enthalpy of reaction for selected vanadium oxide phases tabulated in Refs. 48, 49. Because entropy data were not available for all of these phases, $\Delta S$ of the reactions was assumed to be zero. All lines represent thermodynamic phase equilibria of the oxidation reaction $\mathrm{VO}_{x(s)}+(y / 2) \mathrm{O}_{2(g)} \rightleftharpoons \mathrm{VO}_{x+y(s)}$, where higher temperature and lower oxygen partial pressure stabilizes a vanadium oxide phase with a lower vanadium oxidation state. While $\mathrm{V}_{2} \mathrm{O}_{5}$ is the thermodynamically stable phase at ambient condition, thin-film deposition in vacuum and at elevated temperature are ideally suited to stabilize the desired $\mathrm{VO}_{2}$ phase. Notably, with sample temperatures typically ranging between 300 and $600{ }^{\circ} \mathrm{C}$, growth by pulsed-laser depostion (PLD) in varying oxygen partial pressures has shown that a minimum background oxygen pressure of $10^{-2}$ Torr is required to avoid oxygen deficient $\mathrm{VO}_{2}$ on sapphire substrates. ${ }^{[42]}$ Similarly, for $\mathrm{VO}_{2}$ grown by molecular-beam epitaxy (MBE) distilled ozone background pressures of $10^{-6}$ Torr with substrate temperatures in the $200-350^{\circ} \mathrm{C}$ range, ${ }^{[50-52]}$ or an oxygen plasma pressure ${ }^{[53]}$ of $(1-3) \times 10^{-5}$ Torr at a substrate temperature of $550{ }^{\circ} \mathrm{C}$ were used. ${ }^{[52,53]}$ According to the Ellingham diagram in Fig. 4(b) in all cases $\mathrm{V}_{2} \mathrm{O}_{5}$ is expected to form. The discrepancy between thermodynamic equillibrium calculations and experimental conditions used for the growth of high-quality $\mathrm{VO}_{2}$ films suggests that the growth kinetics play an important role as well. A kinetic barrier to the oxidation of vanadium at the low growth temperatures used to form $\mathrm{VO}_{2}$ films has been observed in surface science experiments, ${ }^{[54]}$ necessitating oxidation conditions that fall in the $\mathrm{V}_{2} \mathrm{O}_{5}$ region of the Ellingham diagram for all growth techniques. Indeed, considering the growth conditions common to $\mathrm{VO}_{2}$ films, it is plausible that some $\mathrm{V}_{2} \mathrm{O}_{5}$ does form during growth, but desorbs due to its high volatility ${ }^{[55]}$ leaving behind a phase-pure film of $\mathrm{VO}_{2}$ in conditions that thermodynamics indicates should be in the territory of $\mathrm{V}_{2} \mathrm{O}_{5}$. Growth at high substrate temperature to overcome the kinetic barrier to oxidation is not an option for two reasons: (1) the rapid interdiffusion of $\mathrm{VO}_{2}$ into the underlying substrates ${ }^{[50,52,54]}$ and (2) if the entire film is oxidized to $\mathrm{V}_{2} \mathrm{O}_{5}$, it will all evaporate due to the high vapor pressure of $\mathrm{V}_{2} \mathrm{O}_{5}{ }^{[55]}$

Despite the many challenges involved in the growth of $\mathrm{VO}_{2}$ films, significant advances have been achieved in its deposition using PLD, ${ }^{[42,56-63]} \mathrm{MBE},{ }^{[52,53,64,65]}$ sputtering, ${ }^{[66-73]}$ electronbeam deposition, ${ }^{[74]}$ ion-beam depostion, ${ }^{[75]}$ metalorganic 
chemical vapor deposition (MOCVD),${ }^{[61,76]}$ chemical solution deposition ${ }^{[77]}$ and atomic layer deposition (ALD). ${ }^{[78,79]}$ Using the resistivity ratio across the MIT as a benchmark for film quality, Fig. 5 shows a comparison of $\mathrm{VO}_{2}$ films grown by different techniques from the aforementioned references. Since strain affects the resistivity ratio across the MIT as well, only fully relaxed films grown on sapphire substrates were selected here. Two general trends are observed: (1) For thick films well above $100 \mathrm{~nm}$, high-quality $\mathrm{VO}_{2}$ films with MIT ratio more than four orders of magnitude are commonly achieved. (2) With decreasing film thickness, the MIT ratios deteriorate to less than two orders of magnitude. Interestingly, recent progress in PLD has demonstrated high-quality $\mathrm{VO}_{2}$ films with an MIT ratio: $>5000$ at film thicknesses as small as $\sim 20$ $\mathrm{nm} \cdot{ }^{[42]}$ Also, growth of high quality $\mathrm{VO}_{2}$ thin films on large silicon wafers has been demonstrated using sputtering. ${ }^{[80]}$ In addition, a combinatorial approach for the growth of $\mathrm{VO}_{2}$ has been developed using a thermal source for $\mathrm{V}$ and the metalorganic precursor vanadium-oxo-triisopropoxide to supply vanadium in a much higher valence state, which demonstrated the potential to combine high-quality $\mathrm{VO}_{2}$ at a dramatically reduced film thickness on wafer scale, while keeping a high resistivity change across the MIT intact. ${ }^{[52-54,64]}$ High-quality $\mathrm{VO}_{2}$ films are a key enabler for electrical engineers to exploit the opportunities offered by electronic PCMs in general and $\mathrm{VO}_{2}$ in particular in proof-of-principle photonic and electronic devices, which are described in detail next.

\section{Augmenting field effect transistors (FETs) with $\mathrm{VO}_{2}$}

In CMOS technology, the backbone of the current computing hardware, the strategy to realize low-power, high-performance computation is to reduce the total energy necessary to switch a FET from an OFF-state to an ON-state. The FET is switched by changing the gate voltage $V_{\mathrm{GS}}$ to modulate the drain-to-source current $I_{\mathrm{DS}}$ by orders of magnitude. The associated energy contains a dynamic contribution $E_{\mathrm{dyn}}=(\alpha / 2) C V_{d d}^{2}$, with the intrinsic transistor gate capacitance $C$ charged to turn the device



Figure 5. Change in resistivity across the MIT, $\Delta \rho / \rho$ (indicated by various symbol) reported for completely relaxed $\mathrm{VO}_{2}$ films grown on sapphire with different thickness. ${ }^{[42,52,53,56-76]}$ The dotted line indicates the thickness at which $\mathrm{VO}_{2}$ is completely relaxed. The data are grouped into the different thin-film deposition techniques, arranged from left to right with increasing scalability. Note that in general a larger film thickness results in a larger $\Delta \rho / \rho$. into the ON state, the supply voltage $V_{d d}$, and dimensionless activity factor $\alpha$ to account for the fraction of clock cycles the transistor is switched. The static contribution is given by $E_{\text {stat }}=I_{\text {leak }} V_{d d} \tau$, with $I_{\text {leak }}$ the leakage current between source and drain in the OFF-state and $\tau$ the switching period, i.e., the clock speed of the processor. Switching the transistor from OFF to $\mathrm{ON}$ is achieved by changing the gate voltage, and along with that the surface potential and band bending $\Psi_{\mathrm{S}}$ in the channel, reducing the energy barrier between source and channel thus modulating the source-drain current $I_{\mathrm{DS}}$.

Even in the OFF-state thermally excited carriers can overcome this barrier, captured by the Boltzmann factor $\exp ^{-\Delta k_{\mathrm{B}}{ }^{T}}$ with $k_{\mathrm{B}}$ the Boltzmann constant, $T$ the absolute temperature, and $\Delta$ the barrier height relative to the Fermi level. The resulting energy dissipation is approximately $E_{\text {stat }}=E_{\text {leak }} \propto V_{d d} e^{-V_{d d} S}$ with $S$ is the subthreshold slope. ${ }^{[81]}$ To ideally balance both contributions to the switching energy per cycle an optimal supply voltage $V_{d d}$, typically close to the transistor threshold voltage is chosen; see Fig. 6. While the transistor would suffer energy losses due to the high leakage energy $E_{\text {stat }}$ for too small operation voltages $V_{d d}$, too high $V_{d d}$ values would unnecessarily increase the dynamic contribution $E_{\text {dyn }}$.

Aside from the supply voltage, another key parameter of transistor performance is the subthreshold slope $S$. It describes the required change in gate voltage to achieve an order of magnitude change in the source-drain current ${ }^{[82]}$

$$
S=\frac{\partial V_{\mathrm{GS}}}{\partial\left(\log I_{\mathrm{DS}}\right)}=\frac{\partial V_{\mathrm{GS}}}{\partial \psi_{\mathrm{S}}} \cdot \frac{\partial \psi_{\mathrm{S}}}{\partial\left(\log I_{\mathrm{DS}}\right)}=\eta \cdot v
$$

which can be separated into the two contributions $\eta$ and $v$ : The body factor $\eta$ describes the coupling of the gate voltage $V_{\mathrm{GS}}$ to the band bending $\Psi_{\mathrm{S}}$ in the channel $\eta=\partial V_{\mathrm{GS}} / \partial \psi_{\mathrm{S}}=1+\left(C_{s} / C_{o x}\right)$, with $C_{s}$ and $C_{o x}$ the semiconductor and gate oxide capacitances, respectively. The transport factor $v$ relates band bending $\Psi_{\mathrm{S}}$ to


Figure 6. (a) Energy per cycle and (b) source-drain current $I_{D S}$ versus gate voltage $V_{G S}$ in units of the supply voltage $V_{d d}$ for transistors with two different subthreshold slopes $S$ of $60 \mathrm{meV} / \mathrm{dec}$. (limit of the Boltzmann switch), and strongly correlated Landau switch with $S=30 \mathrm{meV} / \mathrm{dec}$. 
the source-drain current $I_{\mathrm{DS}}$. In conventional semiconductor materials, the transport factor $v$ is intrinsically limited by the carrier statistics through the Boltzmann factor $10^{-\Delta k_{\mathrm{B}}^{T}}$. Modulating the source-drain current through changes in the gate voltage entails the subthreshold slope as conversion factor $I_{\mathrm{DS}} \sim 10^{-\Delta / k_{\mathrm{B}} T} \sim 10^{-\psi_{\mathrm{S}} / S}$ with subthreshold slope $S \geq\left(q / k_{\mathrm{B}} T\right)$, where $q$ is the elemental charge. In other words, a minimum requirement of $\sim 60 \mathrm{mV}$ change in $\Psi_{S}$ is needed to modulate the source-drain current at room temperature by one order of magnitude: the famous $60 \mathrm{mV} /$ decade limit of conventional FETs. ${ }^{[83]}$ This further implies that for a suitably large distinction between the OFF-state and the ON-state current, the supply voltage must be several times this limit. Consequently, the Boltzmann factor constrains supply voltage scaling, and, therefore, the switching energy of a conventional FET.

Reducing the operating voltage and energy while maintaining performance, i.e., the switching delay and a specific ON/OFF ratio, transistors with a subthreshold slope $<60 \mathrm{mV} /$ decade, referred to as steep-slope transistors, ${ }^{[81,84,85]}$ is therefore demanded. To achieve a subthreshold slope $S<60 \mathrm{mV} /$ decade with $\eta<1$ the integration of a ferroelectric layer into the gate stack to augment the linear gate dielectric has been proposed and is currently being explored. ${ }^{[86-90]}$ The ferroelectric capacitor in series with the dielectric gate capacitor is designed to stabilize an otherwise energetically disfavored reversal pathway that entails an overall negative gate oxide capacitance effect, reversing the sign of $C_{o x}$ and resulting in a body factor, $\eta$, smaller than unity.

Another approach to achieve subthreshold slopes smaller than $60 \mathrm{mV} / \mathrm{decade}$ is to harness transport phenomena to directly beat the Boltzmann limit, hence improving the transport factor $v$. Within the realm of conventional semiconductors, interband tunneling across semiconductor heterojunction [tunnel field effect transistor (TFETs) ${ }^{[91]}$ and carrier multiplication via impact ionization ${ }^{[22,93]}$ in metal oxide semiconductor structures (I-MOS) offer opportunities toward steep-slope switching. The I-MOS concept relies on impact ionization in an ungated intrinsic region between the source region and the channel to induce carrier amplification, which subsequently enables a larger change in current in comparison with conventional CMOS transistors for a given change in gate voltage. Scaling the supply voltage to reduce the energy per cycle, however, is not possible for I-MOS, which along with its disadvantages, namely dead region in the output region precluding inverter operation, reliability issues due to hot carrier formation potentially damaging the dielectric, renders this design unsuitable for high-performance low-power transistors. TFETs utilize a gate tunable tunnel junction in which the conduction and valence band alignment across the heterojunction is modulated, which results in steep turn-ON characteristics for carriers entering the channel from the source. ${ }^{[81,94]}$ Relying on tunneling phenomenon as injection mechanism limits the source-drain current $I_{\mathrm{DS}}$ in the $\mathrm{ON}$ state and thus its suitability for high-performance computing where high $\mathrm{ON}$ currents are required.
Nevertheless, the modulation of the band alignments at the heterojunction is a powerful concept, which suggests an alternative pathway to overcome the Boltzmann limit by employing materials exhibiting electronic phase transitions, as schematically shown in Fig. 7. In contrast to conventional semiconductors, where the electronic bands are only shifted with respect to the Fermi level by an electric field, carriers in correlated electron system can change their character from localized to itinerant and the entire band structure is altered in the vicinity of the Fermi level. Rather than a Boltzmann switch in case of conventional FETs, the concept is best coined as a "Landau switch" in the sense that switching characteristics is not governed by carrier statistics anymore, but rather by the free energy of the competing phases with different electronic ground states. Materials exhibiting strong correlations are therefore uniquely suited to overcome the intrinsic limit of conventional Boltzmann switches by harnessing electronic phase transition. The external perturbation triggering the electronic phase transformation between different ground states does not necessarily have to be an electric field, but could be strain, magnetic field, optical excitation, or other thermodynamic variables, such as temperature or pressure, ${ }^{[95,96]}$ all of those offering novel opportunities toward steep-slope-transistor functionalities.

The Mott-FET ${ }^{[97-100]}$ is a concept in which the semiconducting channel of a conventional transistor is replaced by a phase transition material, for example $\mathrm{VO}_{2}$. The operating principles are as follows: the transistor is initially OFF when the phase transition material is in the high-resistance insulating state. When a sufficient gate field is applied accumulation of free carriers at the dielectric/Mott-material interface screens the Coulomb repulsion among the localized carriers in the insulating state of $\mathrm{VO}_{2}$. Consequently, the electric field triggers an abrupt carrier delocalization inducing the low resistance metallic state. The abrupt liberation of all carriers previously localized to atomic sites enables the transistor to exhibit a sharp turn-on characteristics with $S<60 \mathrm{mV} /$ decade. Attempts to reverse the phase transition by applying an electric field to recapture the insulating ground state and to turn the transistor OFF only had limited success. The external field cannot effectively penetrate into the channel material with metallic-like carrier concentration due to the short Angstrom-scale screening length. ${ }^{[101]}$ The switching has to be bidirectional. The combination of ultrathin films und large field strengths using an ionic liquid gate exhibited very slow switching characteristics, ${ }^{[41,102]}$ hinting that unintentional ionic exchange reactions affecting the channel stoichiometry might be at play as well. ${ }^{[42]}$ The straight forward strategy of a Mott-FET to simply replace the semiconductor material in the channel with a strongly correlated material exhibiting an MIT is thwarted by these roadblocks.

To nevertheless harness the abrupt changes of electronic phase transition materials (EPTMs) while circumventing the field effect requirement, the concept of the Hyper-FET has been proposed and demonstrated using $\mathrm{VO}_{2} \cdot{ }^{[103]}$ Rather than using the MIT material in the channel, the conventional 
Boltzmann switch
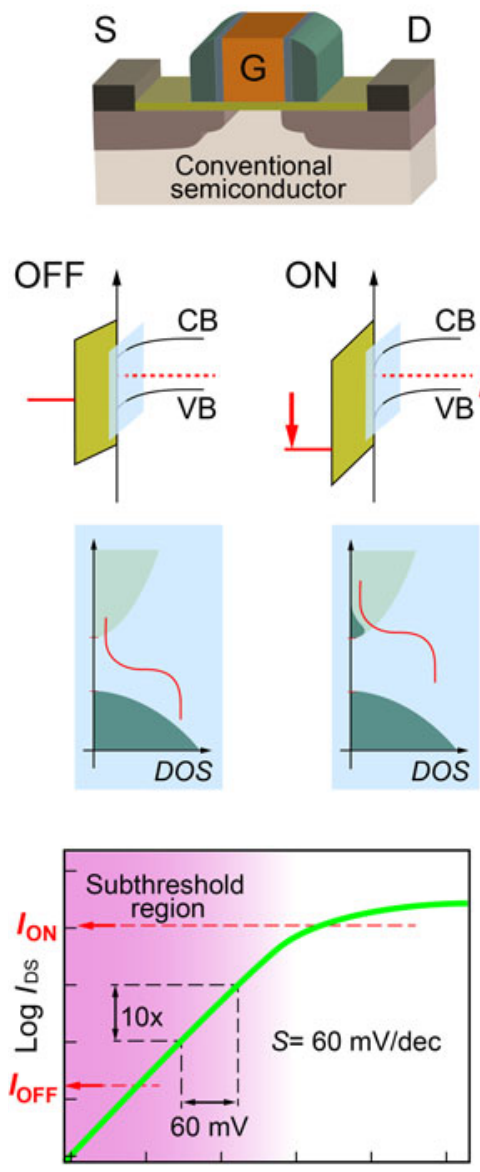

Gate bias
Landau switch
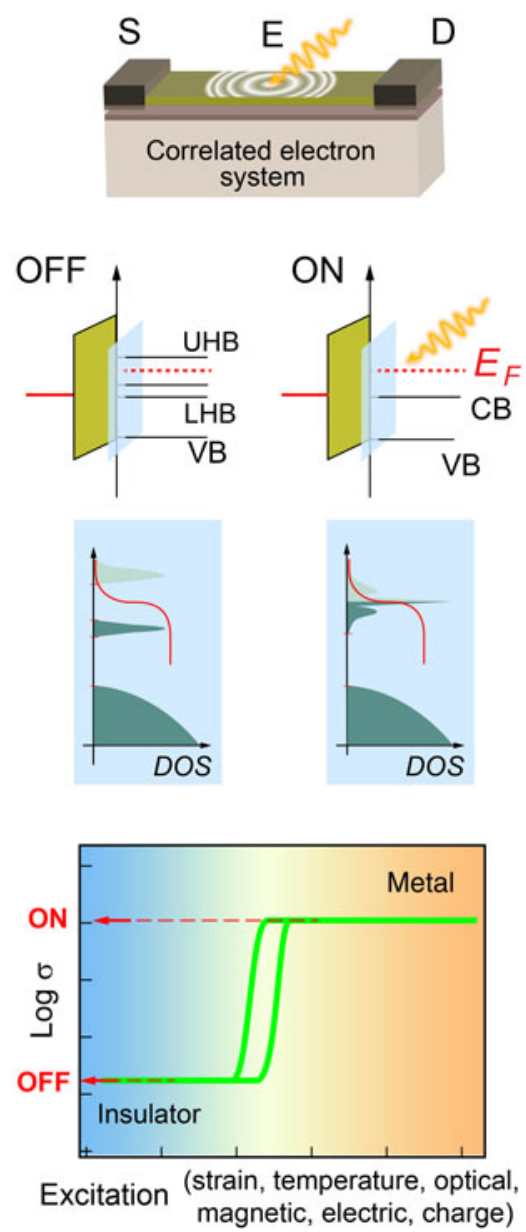

Figure 7. A comparison of the properties of the currently employed Boltzmann switch as FETs (left), which switch state by changing the Fermi level $E_{\mathrm{F}}$ relative to the band structure unaltered by the electric field. The concept of a Landau switch is shown on the right, which is envisioned to operate by changing the DOS at the Fermi level by triggering an electronic phase transition through various external stimulations. Such functionality could overcome the $60 \mathrm{mV} / \mathrm{dec}$. limit intrinsic to conventional FETs semiconductors, enabling low-power, high-performance computing with ultrascaled supply voltages.

FET is augmented by integrating $\mathrm{VO}_{2}$ directly into the source of the conventional MOSFET, as schematically shown in Fig. 8(a). The device design of the Hyper-FET concept ideally integrates abrupt switching phenomenon of $\mathrm{VO}_{2}$ with the superior transport characteristics of a semiconductor channel material, while circumventing the problem of bidirectional electric field switching of $\mathrm{VO}_{2}$. The operation of the Hyper-FET can be described as follows. Initially, at gate voltage $V_{\mathrm{GS}}=0 \mathrm{~V}$, the transistor is in the OFF state with a small channel current $I_{\mathrm{DS}}$ that is further reduced by the high resistance state of $\mathrm{VO}_{2}$. Increasing gate voltage as $V_{\mathrm{GS}}$ increases $I_{\mathrm{DS}}$ until a critical value is reached, which switches $\mathrm{VO}_{2}$ into the metallic state, thereby lowering the series resistance and abruptly increasing $I_{\mathrm{DS}}$; see Fig. 8(b). Similarly, as $V_{\mathrm{GS}}$ is reduced, the channel current decreases until a lower critical current is reached at which $\mathrm{VO}_{2}$ transitions back into the high resistance state. The Hyper-FET exhibits a feedback enhanced gate field controlled abrupt switching characteristics due to the phase transition in $\mathrm{VO}_{2}$ with the switching slope of $12 \mathrm{mV} /$ decade at room temperature. ${ }^{[103]}$ The steep switching slope enables this hybrid phase-transition-FET to achieve higher ON current at matched OFF currents in comparison with conventional MOSFETs. The Hyper-FET primarily enables steep slope operation by achieving a transport factor $v=\left[\partial \psi_{\mathrm{S}}\right]$ $\left.\partial\left(\log I_{\mathrm{DS}}\right)\right]$ less than $60 \mathrm{mV} /$ decade at room temperature due to orders of magnitude abrupt change in resistance in the source, and, thus, providing a much larger current change $\partial\left(\log I_{\mathrm{DS}}\right)$ for an infinitesimally small increment in the channel potential $\partial \psi_{\mathrm{s}}$.

The EPTM integrated into the source of the FET is not restricted to $\mathrm{VO}_{2}$, the device design is scalable and has also been demonstrated using $\operatorname{In}_{0.7} \mathrm{Ga}_{0.3}$ As quantum-well multichannel FinFETs for $n$-MOS and complimentary $p$-MOS using a Ge quantum-well multi-channel FinFETs. ${ }^{[103]}$ 
(a)
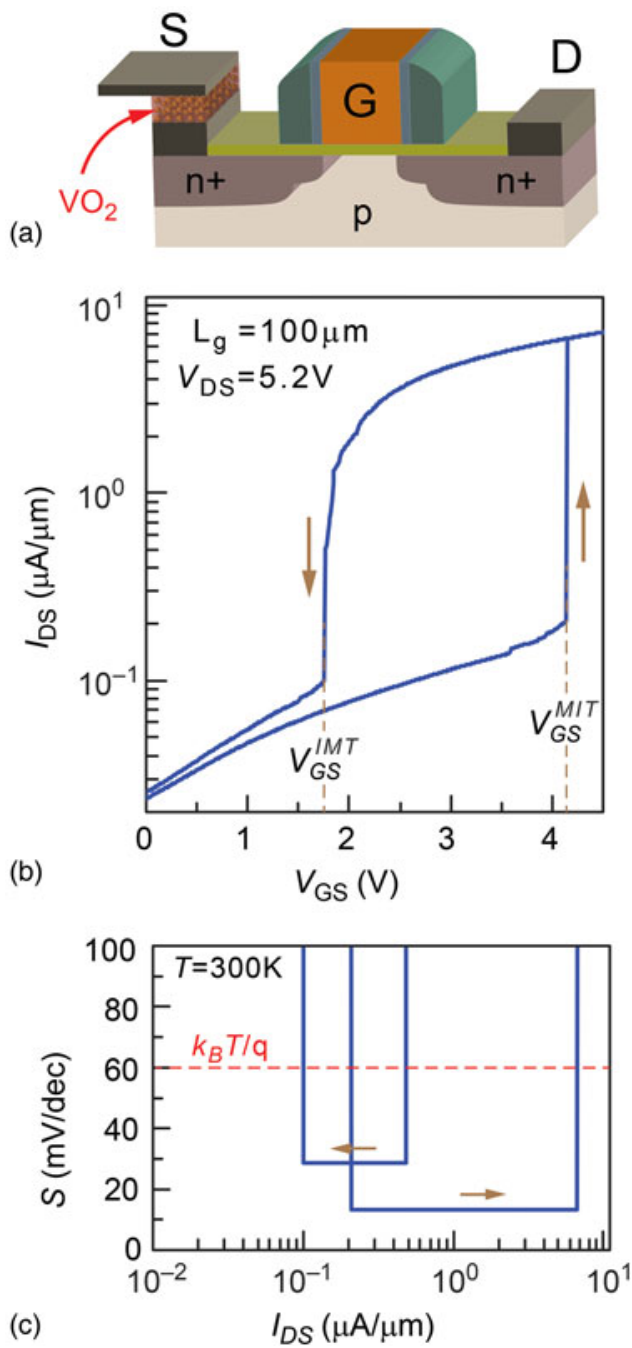

Figure 8. (a) Schematic representation of the Hyper-FET wherein an electronic PCM exhibiting an electronically induced abrupt change in resistivity (such as $\mathrm{VO}_{2}$ ) is integrated in series with the source of a conventional MOSFET. (b) Transfer characteristics $\left(I_{D S}-V_{G S}\right)$ of the $\mathrm{VO}_{2}$-based Hyper-FET at room temperature. (c) Switching slope $S$ of the Hyper-FET as a function of drain-to-source current $I_{D S}$ demonstrating a sub- $k_{\mathrm{B}} T / q$ switching-slope (i.e., $<60 \mathrm{mV} / \mathrm{dec}$ ) at room temperature [panels (a) and (b) are adapted from Ref. 103].

While proof-of-principle has been demonstrated, technological challenges to implement this device concept into mainstream technology remains to be addressed. First, it needs to be demonstrated that aggressive scaling of the Hyper-FET design to below $10 \mathrm{~nm}$ can be achieved with maintaining a sizeable, (i.e., few orders of magnitude) resistivity change across the MIT of $\mathrm{VO}_{2}$; this then favorably reflects into transfer characteristics with much larger source-drain current for a fixed, small gate voltage modulation. Further, given that the MIT in $\mathrm{VO}_{2}$ is affected by temperature, a potential sensitivity of transfer characteristics to the chip temperature when implemented in portable electronics, and more under heavy computation loads when large amounts of heat are being dissipated, are additional practical concerns to be addressed in the future. While $\mathrm{VO}_{2}$ might not be an ideal materials solution for the application, the task for material scientists will be to find a better suited electronic PCM that allows for scaling and for a more seamless integration into the existing fabrication process. The concept of the Hyper-FET uniquely combines the abrupt collective carrier response from phase transition materials arising from strong electron correlation present in small band width material with the superior carrier transport characteristics in semiconductor materials stemming from the small carrier effective mass enabled by a large band width, while avoiding the need to directly induce the phase transition by an electric field. It therefore represents an ideal symbiosis of the two entirely different material classes of semiconductors and electronic PCMs.

\section{Non-Boolean computing using charge coupled $\mathrm{VO}_{2}$ oscillators}

While in the Hyper-FET concept the MIT material has merely the role to augment, it dramatically improved the conventional FET characteristics dominated by the semiconductor channel by utilizing the abrupt resistivity change of $\mathrm{VO}_{2}$. A completely different aspect of computation, namely associative computing, has been explored and can be potentially harnessed if the attention is focused back on the two-terminal $\mathrm{VO}_{2}$ device as the active element and simplifying the function of the FET in series to that of a tunable resistor $R_{\mathrm{s}}$, as shown in Fig. 9(a). The abrupt and hysteretic evolution of current passing through $\mathrm{VO}_{2}$ as a function of applied voltage encompasses the fundamental instability of the first order phase transition, separating the insulating from the metallic state. In the case of a negligible series resistance $R_{\mathrm{S}}$ the insulator-to-metal transition (IMT), i.e., the transition from the insulating to the metal state of $\mathrm{VO}_{2}$ is triggered at a critical field strength of $E_{2}=V_{2} / L$, determined from the voltage drop $V_{2}$ across $\mathrm{VO}_{2}$ and the electrode distance $L$; see Fig. 9 (b). The applied voltage has to then be reduced to $E_{1}=V_{1} / L$ to reverse the transition, i.e., going from the metallic back to the insulating state (MIT) to obtain the high resistance state again. Small series resistances will merely affect this hysteretic switching behavior. However, the abrupt reduction of the $\mathrm{VO}_{2}$ resistance at the critical field $E_{2}$ will redistribute the voltage drops across the series resistor $R_{\mathrm{S}}$ and the $\mathrm{VO}_{2}$ device. Triggering the metallic state in $\mathrm{VO}_{2}$ results in an instantaneous current increase and reduction of the voltage across the $\mathrm{VO}_{2}$ device, thus acting as a negative differential resistance. For a series resistance above the critical value $R_{\mathrm{C}}$ the change in voltage drop across the $\mathrm{VO}_{2}$ device is so high that the remaining field strength is less than the critical field $E_{1}$ required to stabilize the metallic state. Under these conditions and even though the metallic state was nucleated at the critical field value $E_{2}$, the high resistance state in $\mathrm{VO}_{2}$ cannot be completely annihilated. Rather, the low electric field now favors the insulating state of $\mathrm{VO}_{2}$, reversing the transition. This in turn increases the device resistance, and, with it the voltage drop across $\mathrm{VO}_{2}$ device, again favoring the metallic state. The sufficiently large series 


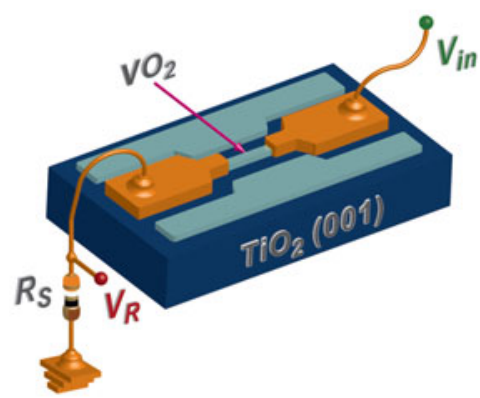

(a)

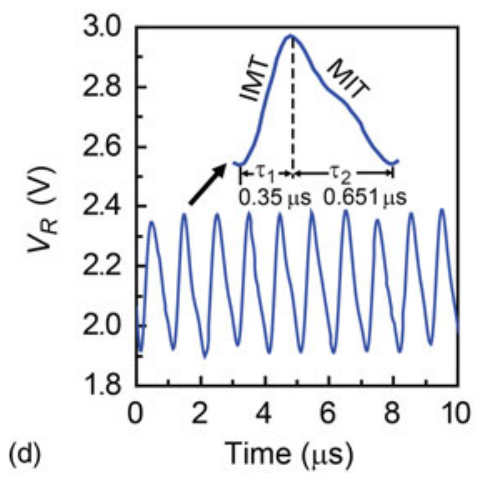

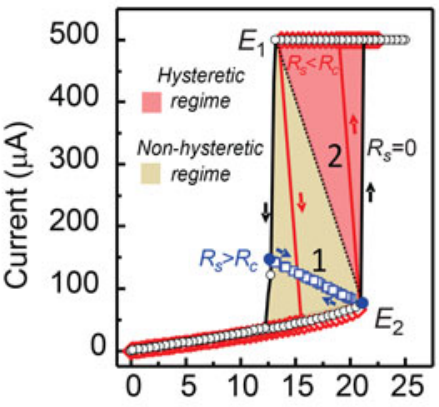

(b) Electric field $(\mathrm{kV} / \mathrm{cm})$



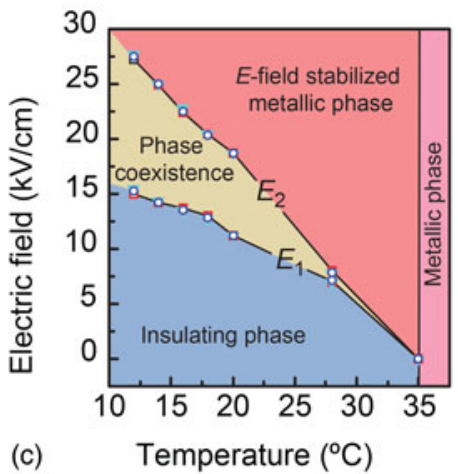

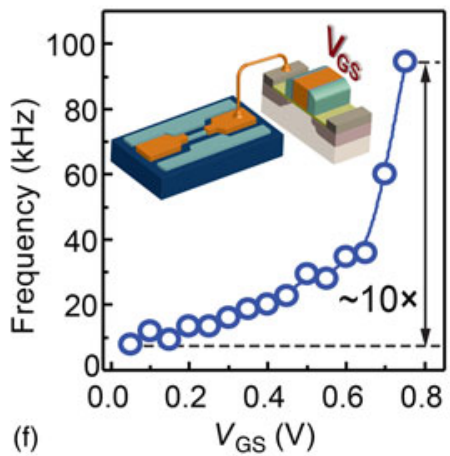

Figure 9. (a) Schematic representation of the $\mathrm{VO}_{2}$-based relaxation oscillator: a two-terminal $\mathrm{VO}_{2}$ device in series with a resistor $R_{\mathrm{S}}$. (b) Current versus voltage characteristics of the $\mathrm{VO}_{2}$ device: no $R_{\mathrm{S}}$ (black), $R_{\mathrm{S}}<R_{\mathrm{C}}$ (red load line 2), and with $R_{\mathrm{S}}>R_{\mathrm{C}}$ (blue load line 1). While hysteretic transition and abrupt change in current is found for $R_{\mathrm{S}}<R_{\mathrm{C}}$, a non-hysteretic transition with oscillations is observed for $R_{\mathrm{S}}>R_{\mathrm{C}}$. (c) Temperature dependence of the critical electric-fields $E_{2}$ and $E_{1}$ for $\mathrm{VO}_{2}$ devices grown on (001) $\mathrm{TiO}_{2}$. An intermediate region of phase coexistence of both, the insulating and metallic phases was accessible, enabling the oscillatory behavior of $\mathrm{VO}_{2}$. (d) Typical time-domain wave form of the $\mathrm{VO}_{2}$-based relaxation oscillator. Inset shows the one oscillation period with the two time constants $\left(\tau_{1}=0.35\right.$ and $\left.\tau_{2}=0.651 \mu \mathrm{s}\right)$ associated with the IMT and the MIT. (e) Effect of input voltage $V_{\text {in }}$ and series resistor $R_{\mathrm{S}}$ on the operating frequency of the oscillator. (f) Frequency versus gate voltage $V_{G S}$ characteristics of a voltage-controlled $\mathrm{VO}_{2}$ oscillator constructed by replacing $R_{\mathrm{S}}$ with a MOSFET; allowing to tune the series resistance using $V_{G S}$ [panels (b), (c), (e), and (f) are adapted from Ref. 184, and (d) is adapted from Ref. 110].

resistance stabilizes $\mathrm{VO}_{2}$ into a non-hysteretic phase coexistence regime, defined within the bounds of the critical field values $E_{2}$ and $E_{1}$, which are strongly temperature dependent; see Fig. 9(c). The waveform of the oscillation induced by a DC signal is shown in Fig. 9(d), which has been measured by probing the voltage drop $V_{R}$ across the series resistor [Fig. 9(a)]. An increase in $V_{\mathrm{R}}$ is found upon decreasing the $\mathrm{VO}_{2}$ resistance (IMT), while a reduction of $V_{\mathrm{R}}$ is associated with a resistance increase of $\mathrm{VO}_{2}$ (MIT). The associated time constants for the IMT and the MIT of $\tau_{1}(0.35 \mu \mathrm{s})$ and $\tau_{2}(0.65 \mu \mathrm{s})$, respectively, were dominated by parasitic resistances and capacitances present in the simple experimental setup, and thus orders of magnitude larger than the intrinsic time constants of the $\mathrm{VO}_{2}$ phase transition. ${ }^{[104]}$ Reducing the series resistance $R_{\mathrm{S}}$ increased the frequency of the $\mathrm{VO}_{2}$ oscillation, Fig. 9(e). A continuous change in the $R_{\mathrm{S}}$ can be achieved by replacing $R_{\mathrm{S}}$ with a transistor and adjusting its channel resistance using the gate voltage $V_{\mathrm{GS}}$, Fig. 9(f).

Coupling these highly scalable, inductor-free voltage controlled charge oscillator devices and investigating the synchronization dynamics of those coupled devices could open up opportunities to realize a simple hardware platform for a specific class of computing problems, referred to as associative computing. ${ }^{[105]}$ Compared with sequential algorithms developed for conventional CMOS hardware the synchronization dynamics of coupled oscillators, an inherently parallel process, could be potentially used to solve computationally hard problems more efficiently. Such tasks range from combinatorial optimization problems like graph coloring ${ }^{[106]}$ or the traveling salesman problem, ${ }^{[107]}$ and include associative computating problems related to image and pattern recognition, ${ }^{[108-110]}$ as well as scene segmentation and saliency detection problems using the concept of oscillatory locally excitatory globally inhibitory networks ${ }^{[111]}$ required in computer vision systems.

To investigate the coupling behavior of $\mathrm{VO}_{2}$ oscillators, two devices were stabilized at different frequencies of 48.43 and $37.27 \mathrm{kHz}$ by choosing different values of $R_{\mathrm{S}}$ (38 and 47 $\mathrm{k} \Omega$ ). Figure 10(a) schematically shows how both elements were linked using a capacitor $\left(C_{\mathrm{C}}=680 \mathrm{pF}\right)$ as the coupling element. The different operating frequencies of each oscillator prior to coupling shown in Fig. 10(b) indicated that the oscillators were completely independent. After pairing them with the 
coupling capacitor, the oscillators synchronized to a common resonant frequency. A narrowing of the spectral line width further corroborated the locking of the two oscillators and the presence of a mutual feedback stabilizing the synchronized oscillator pair against noise. Figure 10(c) shows the time evolution of the coupled resistance states of both oscillators, 1 and 2. Tracing the trajectory in the phase diagram by using the voltage drops $V_{\mathrm{R}, 1}$ and $V_{\mathrm{R}, 2}$ across both series resistances $R_{\mathrm{S}, 1}$ and $R_{\mathrm{S}, 2}$ allowed one to determine the metallicto-insulating phase ratio in the individual devices. As device-1 transitioned from the insulating toward the metallic phase, device-2 changed from the metallic to the insulating state [Section "Strong electron correlations in V-based oxides" of the phase space trajectory in Fig. 10(c)]. Conversely, when device-1 was in a more metallic and device- 2 was in a more insulating state, charge was exchanged through the coupling capacitance and moved from device-1 to device-2, represented by the Section "Ternary oxide vanadates" of the trajectory in Fig. 10(c).

The precise shape of phase space trajectories of the coupled oscillator pair has been found strongly dependent on the difference of the series resistances $R_{\mathrm{S}, 1}$ and $R_{\mathrm{S}, 2}$. Therefore, as shown in Fig. 10(d), replacing the resistors by conventional MOSFETs and using the gate voltages $V_{\mathrm{GS}, 1}$ and $V_{\mathrm{GS}, 2}$ as input parameter, their degree of match is decoded into a specific shape of the trajectory in the $V_{R, 1}-V_{R, 2}$ phase space. While for large differences in the input voltages $V_{\mathrm{GS}, 1}$ and $V_{\mathrm{GS}, 2}$ no stable trajectory was formed - the oscillator pair did not sychronize - smaller differences $\Delta V=V_{\mathrm{GS}, 2}-V_{\mathrm{GS}, 1}$ dramatically altered the trajectory, as schematically depicted in Fig. 10(e). To analyze these changes and form a simple output value from the coupled oscillator pair an average exclusive-OR (XOR) measure applied to $V_{\mathrm{R}, 1}$ and $V_{\mathrm{R}, 2}$ was used; a time-equivalent metric to quantify how long the coupled oscillators remained in the gray-shaded area in phase space during an oscillation period, as indicated in Fig. 10(e). While the XOR values were small for $\Delta V \approx 0$, the nonlinear increase with $\Delta V$ shown in Fig. 10(f) closely resembled the Euclidean norm $L_{1 / 2}=\left(X_{1}^{2}+X_{2}^{2}\right)^{1 / 2}$ shown in Fig. $10(\mathrm{~g})$.

This sensitivity of the coupled oscillators' phase space orbit to the difference in the input parameters forms the basic computational hardware building block of the coupled oscillators. A non-linear distance norm ${ }^{[12]}$ that can be used to determine how closely the two analog voltage input values resemble each other is realized by the simple hardware building block of capacitively coupled two-terminal electronic PCM, allowing for fast and energy-efficient computation of tailored non-linear degree-of-match-type analysis. ${ }^{[110,112]}$ This task will play a critical role in the paradigm shift from precise computing on small data sets to approximate computing on large data sets, which has already occurred in future application areas, such as machine learning, augmented reality, and data base search. ${ }^{[113]}$ A dramatically increasing demand to solve this task elegantly in a fast, computationally inexpensive, and thus, energetically efficient way, is projected for data analysis generated from massive sensor arrays in the dawning IOT (internet-of-things) era.
Two-terminal devices made from electronic PCM exhibiting current-controlled resistive switching have been identified as key in neuristors ${ }^{[114]}$ to mimic biological functionality of neural networks using solid-state electronic circuitry, a further evidence of the critical role of these types of materials to meet future computation demands.

\section{$\mathrm{VO}_{2}$ as $\mathrm{RF}$ switch}

Currently state-of-the-art switches can be categorized into MEMS and solid-state devices, both of which enable switching between a high-impedance (OFF) state and a low-impedance (ON) state for RF frequency signals. One of the key performance parameter of an RF switch is the cut-off frequency, $f_{\mathrm{CO}}$, at which the ratio of impedance of the ON state and OFF state is unity, thus defining the maximum operating frequency

$$
f_{\mathrm{CO}}=\left(2 \pi R_{\mathrm{ON}} C_{\mathrm{OFF}}\right)^{-1}
$$

with $R_{\mathrm{ON}}$ is the ON state resistance, and $C_{\mathrm{OFF}}$ is the OFF state capacitance.While solid-state semiconductor-based RF switches are limited to $f_{\mathrm{CO}}$ at least 2-3 orders lower than MEMS switches due to their comparably high $R_{\mathrm{ON}}$ and high $C_{\mathrm{OFF}}$, their low drive voltage, high switching speed, and excellent reliability due to no moving mechanical parts render them a highly competitive solution. Near zero power consumption, high electrical isolation in the OFF state, very low insertion losses, high cut-off frequencies, and highly linear performance make MEMS switches attractive, compensating for their low switching speeds, high voltage, or current drive needed to actuate the switch despite their higher fabrication costs and lower reliability. The ideal RF switch would be a combination of both approaches, a simple solid-state design exhibiting a fast-switching characteristics along with a MEMS-like high frequency of operation and low insertion loss.

One approach to realize such an RF switch is to harness the change in resistivity across the MIT in TMOs such as $\mathrm{VO}_{2}$. Demonstrations and benchmarking of $\mathrm{VO}_{2}$-based RF switches have been done using films grown by pulse laser deposited $(\mathrm{PLD})^{[115]}$ and sputtering. ${ }^{[116]}$ While these deposition techniques produce films with varying degree of crystallinity and strain, a relatively low $R_{\mathrm{OFF}} / R_{\mathrm{ON}}$ ratio and increase in device capacitance due to grain boundaries causing undesirable parasitic contributions and reducing $C_{\mathrm{OFF}}$ are expected to detrimentally affect device performance. For the best performance single-crystalline $\mathrm{VO}_{2}$ films with a large change in the resistivity across the MIT grown on a low loss substrate using a scalable deposition technique is desired. Figure 11(a) shows a false-colored secondary electron micrograph of a solid-state RF switch. A ground-signal-ground (GSG) pad structure consisting of 24 parallel two-terminal $\mathrm{VO}_{2}$ devices with $1 \mu \mathrm{m}$ width and $100 \mathrm{~nm}$ length was fabricated from a 30-nm-thick single-crystalline $\mathrm{VO}_{2}$ film with resistivity ratios larger than $10^{4}$ grown on sapphire using a combinatorial growth approach. ${ }^{[64]}$ Similar to the Hyper-FET concept, the transition to the low impedance metallic state was triggered by applying 

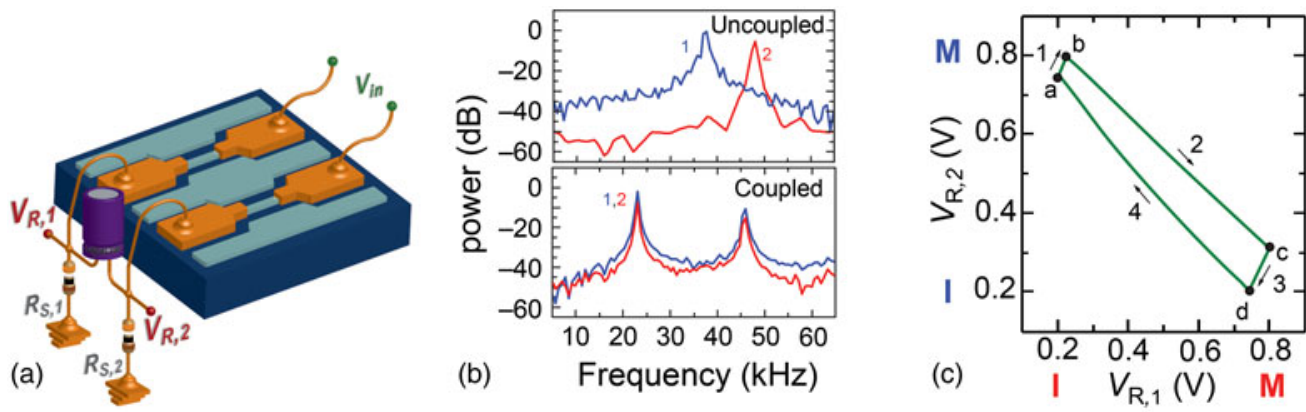

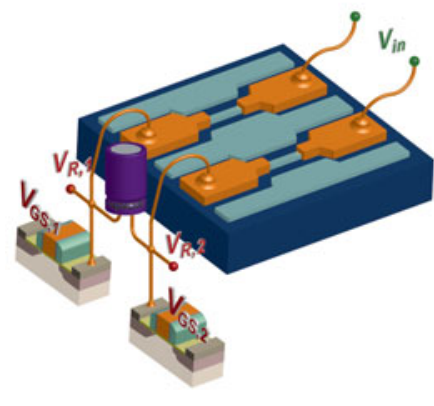

(d)
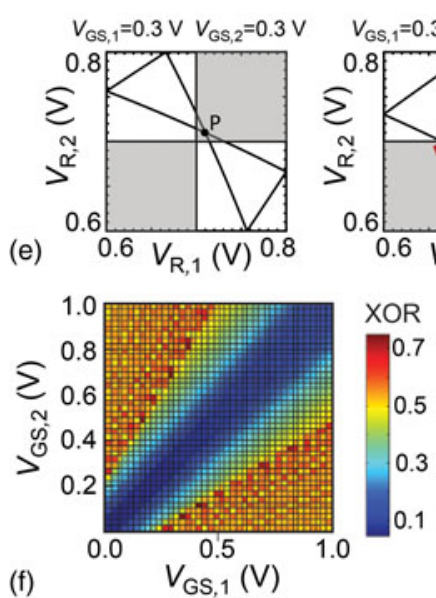
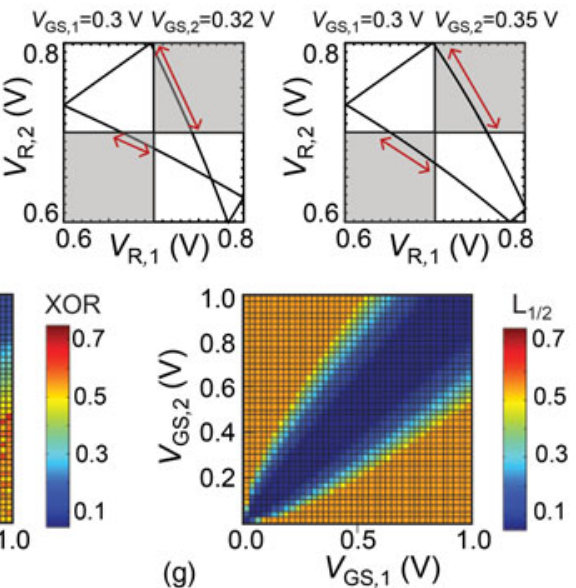

Figure 10. (a) Schematic of the capacitively coupled $\mathrm{VO}_{2}$ oscillators. (b) Spectral characteristics of the oscillators before and after capacitive coupling. The individual oscillators synchronize to a common resonant frequency when coupled. (c) Simulated phase space trajectory of the coupled oscillators. The evolution of phase composition in the coupled $\mathrm{VO}_{2}$ devices is determined from $V_{R, 1}$ and $V_{R, 2}$. Coupled oscillators predominantly show out of phase locking with one oscillator in the metallic state, while the other is in the insulating state. (d) Schematic representation of a pair of voltage tunable coupled $\mathrm{VO}_{2}$ oscillators wherein

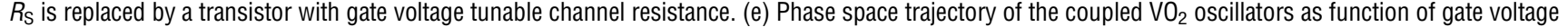
difference $V_{\mathrm{GS}, 2}-V_{\mathrm{GS}, 1}$. As the degree of similarity between the inputs decreases $V_{\mathrm{GS} 2}-V_{\mathrm{GS} 1}$ becomes larger (inputs are similar when $V_{\mathrm{GS}, 2}-V_{\mathrm{GS}, 1}=0$ ) and the oscillators exhibit increased in-phase locking behavior (shaded area in the $V_{R, 1}-V_{R, 2}$ phase space). (f) Simulated time average XOR output of the coupled oscillators, and $(\mathrm{g}) L_{1 / 2}$ distance norm as a function of $V_{\mathrm{GS}, 1}$ and $V_{\mathrm{GS}, 2}$. The qualitative match between the XOR output and the $L_{1 / 2}$ distance norm indicates that the XOR output of the coupled oscillators resembles the nonlinear distance norm of the Euclidean space [panels (b) and (c) are adapted from Ref. 184, and (e)(g) are adapted from 110].

a DC current. A critical current of $5 \mathrm{~mA}$ was needed to destabilize the high impedance state. An attenuation change due to the MIT in $\mathrm{VO}_{2}$ for a $1 \mathrm{GHz}$ signal from $35 \mathrm{~dB}$ in the OFF state $\left(I_{\mathrm{DC}}=0 \mathrm{~mA}\right)$ to $0.55 \mathrm{~dB}$ in the $\mathrm{ON}$ state $\left(I_{\mathrm{DC}}=25 \mathrm{~mA}\right)$ was measured. Figure 11(b) shows the switching response of the device for a $50 \mathrm{MHz}$ RF signal and a $250 \mathrm{~ns}$ pulse of $2 \mathrm{~V}$ amplitude to activate the switch. A fast turn-on characteristic of $25 \mathrm{~ns}$ was found along with an excellent endurance without observing device breakdowns after cycling between $\mathrm{ON}$ and OFF states for more than $10^{8}$ times, shown in Fig. 11(c).

These testaments of fast response and reliability of the $\mathrm{VO}_{2}$-based solid-state RF switch are ideally complemented by a high cut-off frequency of $26.5 \mathrm{THz}$, enabled by the low insertion loss of metallic $\mathrm{VO}_{2}$ in the $\mathrm{ON}$ state and a large OFF state capacitance due to excellent microstructural properties of the grown $\mathrm{VO}_{2}$ films. Compared with a state-of-the-art GaN based solid-state RF switch using a superlattice structure with a castellated three-dimensional (3D) gate $^{[117]}$ a $10 \mathrm{~dB}$ improvement in the switching ratios for a $20 \mathrm{GHz}$ signal highlights the potential of $\mathrm{VO}_{2}$-based $\mathrm{RF}$ switches for highfrequency signal operation, outperforming conventional solidstate FET designs, and III-V-based high-electron-mobility transistors (HEMTs). ${ }^{[118]}$ Figure 11(d) compiles cut-off frequencies of several MEMs and solid-state RF switching technologies with PCM and $\mathrm{VO}_{2}{ }^{[119-126]}$ While $\mathrm{VO}_{2}$ devices were also operated using local heating, which revealed a higher cut-off frequency of $f_{\mathrm{CO}}=44 \mathrm{THz}$, the turn-on characteristics is expected to be not as competitive due to the necessity to integrate an in-line resistive heater. While the technology for integrated local heater elements have been developed and demonstrated for PCMs such as GeTe, ${ }^{[122]}$ which also suffer from the requirement of a continuous DC power during the $\mathrm{ON}$-state, longer switching times and isolation challenges due to RF leakage through the resistive heater in the OFF state are avertible complications of this design. Thus, EPTMs in general and $\mathrm{VO}_{2}$ on sapphire in particular provide a viable path toward realization of high-performance RF switches, ideally combining the MEMS-like high frequency of operation 


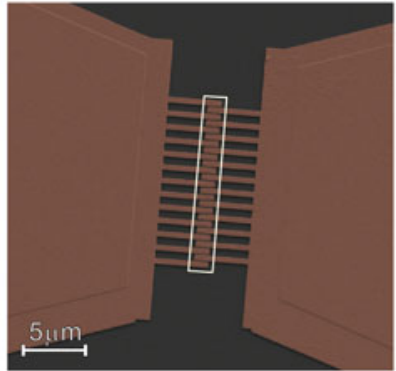

(a)

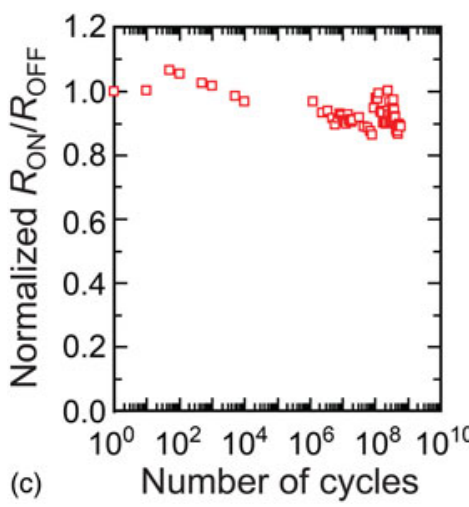

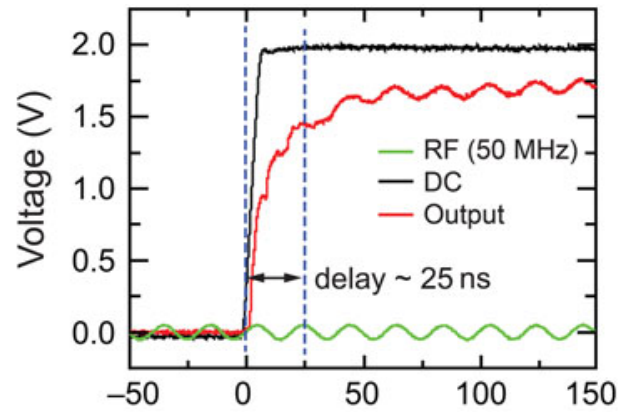

(b)



Figure 11. (a) False-colored scanning electron micrograph (SEM) of a $\mathrm{VO}_{2}$-based solid-state RF switch, a two-terminal GSG structure made up of 24 parallel channels, each with a width of $1 \mu \mathrm{m}$ and length of $100 \mathrm{~nm}$. (b) Turn ON characteristics of the $\mathrm{VO}_{2}$-based RF switch with a DC activating pulse (black) and $50 \mathrm{MHz}$ $\mathrm{RF}$ signal (green). The output pulse (red) shows that the switch can be turned ON in $<25 \mathrm{~ns}$. (c) Endurance test of the solid-state $\mathrm{VO}_{2} \mathrm{RF}$ switch. Stable $R_{\mathrm{ON}} / R_{\mathrm{OFF}}$ ratio (normalized to the first cycle) is observed for close to one billion cycles. (d) Performance benchmarking of various RF switch technologies, comparing the typical OFF-state capacitance $\left(C_{\mathrm{OFF}}\right)$ at a specified ON-state resistance $\left(R_{\mathrm{ON}}\right) ; ;^{[119-126]}$ the color scale represents the cut-off frequency $f_{\mathrm{co}}$. PCMs and EPTMs, such as $\mathrm{VO}_{2}$, ideally combine the superior performance of MEMS with the reliable and fast switching characteristics of solid-state switches [panels (a)-(d) are adapted from Ref. 118].

with reliability and fast switching characteristics of a solid-state design, harnessing the favorable properties of a MIT in this application.

\section{Ternary oxide vanadates}

The previous section (MIT in binary vanadium compounds: $\mathrm{VO}_{2}$ and $\mathrm{V}_{2} \mathrm{O}_{3}$ ) was focused on the binary oxides of vanadium $\mathrm{V}_{x} \mathrm{O}_{y}$ in general and $\mathrm{VO}_{2}$ in particular. Their crystal structure is built from a particular geometrical arrangement of oxygen octahedra coordinating vanadium exhibiting different connectivity. If, however, a third element, such as $\mathrm{Sr}, \mathrm{Ca}$, or a rare-earth cation, is added in a 1:1 stoichiometric ratio to vanadium, the oxygen octahedron connectivity changes from a combination of corner-, edge-, and side-sharing arrangement to the solely corner-sharing $\mathrm{ABO}_{3}$ perovskite structure to make room to accommodate the large $A$-site cation. The perovskite unit cell is shown in Fig. 12, containing a single oxygen ochaedron with oxygen at the face center, the $A$-site at the corners, the $B$-site at the body-center. The perovskite structure can host many combinations of different elements occupying the $A$ and $B$-sites thereby encompassing a large diversity of physical properties. ${ }^{[1,127]}$ Here, we focus on vanadate materials with perovskite structure, i.e., systems in which vanadium occupies the $B$-site. Despite the simple connectivity in this crystal structure, many structural variants from this high-symmetry cubic structure are known, ${ }^{[128-131]}$ which can be thought of as displacements of the cations and tilts, rotation, stretching or compression of the oxygen octahedra without losing the corner connectivity. The small deviations in the $\mathrm{V}-\mathrm{O}-\mathrm{V}$ bond length and bond angle have dramatic effects on the charge, spin, and orbital order and thus the electronic ground state. ${ }^{[132]}$

To describe and understand these structural distortions intuitively Glazer's notation is commonly employed. ${ }^{[128,129]}$ A pseudocubic unit cell is used accommodating rotations of the oxygen octahedra about the three cubic axes with adjacent octahedron either in-phase or out-of-phase. The notation uses a triplet with superscripts to specify lattice parameter and rotation pattern of the oxygen octahedra, respectively. For example, $\mathrm{SrVO}_{3}$ is cubic (space group: $P m \overline{3} m$ ) with $a=b=c=3.842$ $\AA$, i.e., no tilt or rotation, which is described in Glazer's notation as $a^{0} a^{0} a^{0}$; in contrast, $\mathrm{CaVO}_{3}$ and $R \mathrm{VO}_{3}(R$ is a rare-earth element) are both orthorhombically distorted (space group: Pbnm), exhibiting the octahedral distortion pattern $a^{-} a^{-} c^{+}$. Rotations about the $x$ - and $y$-axes are the same and alternate 
$\mathrm{AVO}_{3}$ pseudocubic unit cell


$a^{-}$



$C^{+}$



$a^{-}$

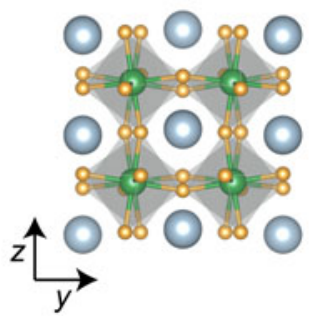

Figure 12. Prototypical crystal structure of the $\mathrm{ABO}_{3}$ perovskite. (a) Cubic unit cell of an undistorted perovskite unit cell, consisting of a single oxygen octahedral unit that coordinates the central V-atom. (b-d) Projections along the pseudocubic axes of an orthorhombically distorted perovskite (space group Pbnm). The symmetry lowering is reflected by a unit cell doubling.

between positive and negative rotations for adjacent octahedra along these axes, indicated by $a^{-}$(see lower panels in Fig. 12). In contrast, the rotation about the $z$-axis is in-phase (see upper right panel, Fig. 12). These subtle structural distortions are important in tuning electron correlation effects in perovskite vanadates.

This section first discusses electron correlation effects in the $d^{1}$ correlated metals $\mathrm{SrVO}_{3}$ and $\mathrm{CaVO}_{3}$, followed by the challenges and recent advances in the growth of thin films of the $A \mathrm{VO}_{3}$ perovskite vanadates. In this context, emphasis is placed on how electron correlations could be further enhanced to drive these metals exhibiting strong electron correlations across the MIT to realize a vanadate based $d^{1}$ Mott-insulating phase. It is then reviewed how electron correlation can be harnessed even far away from any electronic phase transition if the metallic ground state is present. Both correlated metals $\mathrm{SrVO}_{3}$ and $\mathrm{CaVO}_{3}$ are representative of a new class of transparent conducting materials, which are critical components in all current display and photovoltaic technologies. This new class of transparent conductor (TC) is based on a novel design paradigm beyond the conventionally employed scheme to degenerately dope wide band gap semiconductors. Here, rather than increasing carrier concentration, mass enhancement of delocalized carriers in a high-density electron liquid is used to reduce the plasma frequency to below the visible spectrum. ${ }^{[133]}$ Changing the electronic configuration of perovskite vanadates from $d^{1}$ to $d^{2}$ by substituting divalent $\mathrm{Sr}$ and $\mathrm{Ca}$ by trivalent rare-earth ions, the correlation strength is substantially increased with respect to the conduction band width $W$ and a Mott-insulating phase emerges along with interesting coupling

of spin and orbital DOF that is sensitive to structural distortion. $\mathrm{LaVO}_{3}$, a representative of rare-earth vanadates exhibiting a $\sim 1.1 \mathrm{eV}$ Mott gap, will be discussed as a new absorber material for an all oxide solar cell.

\section{Correlation effects in $d^{1}$-filled $\mathrm{SrVO}_{3}$ and $\mathrm{CaVO}_{3}$}

Both $\mathrm{SrVO}_{3}$ and $\mathrm{CaVO}_{3}$ are correlated metals with a nominal $3 d^{1}$ configuration. The strong correlation properties are seen by a reduced band width compared with density functional theory calculations, as measured by angle-resolved photoemission spectroscopy. ${ }^{[134,135]}$ As schematically shown in Fig. 2, the spectral weight transfer feature from the coherent quasi-particle peak to incoherent side bands has been experimentally observed in photoemission experiments, ${ }^{[136-138]}$ a precursor to a Mott-insulating state with a lower and upper Hubbard band separated by a sizeable Mott gap. The $\mathrm{Ca}^{2+}$ ions have a smaller ionic radii $(1.34 \AA)$ than $\mathrm{Sr}^{2+}$ ions (1.44 $\AA$ ) driving the structural distortion from the $a^{0} a^{0} a^{0}$ cubic structure of $\mathrm{SrVO}_{3}$ (with $\left.a=3.842 \AA\right)^{[139]}$ to the $a^{-} a^{-} c^{+}$orthorhombic structure of $\mathrm{CaVO}_{3}$ (with orthorhombic lattice parameters $a=$ 5.326, $b=5.352, c=7.547 \AA$, and pseudocubic lattice constant of $a=3.775 \AA$ ). The orthorhombic distortion reduces the $\mathrm{V}-\mathrm{O}-$ $\mathrm{V}$ bond angle from the ideal $180^{\circ}$ in $\mathrm{SrVO}_{3}$ to $\sim 160^{\circ}$ in $\mathrm{CaVO}_{3}$, accompanied with a reduced orbital overlap between neighboring V $3 d$ orbitals mediated by oxygen $2 p$ orbitals and therefore resulting in a decreased bandwidth $(W)$ by a factor of $\sim \cos ^{2}(\theta)$, i.e., about 6\%. ${ }^{[140,141]} \mathrm{The} \mathrm{Sr}_{x} \mathrm{Ca}_{1-x} \mathrm{VO}_{3}$ solid solution system has been investigated to explore how the structural distortion couples to band width, and thus correlation effects, since this compound interpolates between the $a^{0} a^{0} a^{0}$ and $a^{-} a^{-} c^{+}$structures while maintaining the $3 d^{1}$ electronic configuration. Furthermore, the V $3 d$ bands were well separated from O $2 p$ band, minimizing complex hybridization and thus charge transfer effects. The spectral weight transfer of $\sim 2 \mathrm{eV}$ below the Fermi level was determined from UPS [ultraviolet (UV) photoemission spectroscopy] measurement by systematically decreasing the composition of $\operatorname{Sr}(x)$ in the polycrystalline $\mathrm{Sr}_{x} \mathrm{Ca}_{1-x} \mathrm{VO}_{3}$ system. ${ }^{[142]}$ However, despite the large spectral transfer that confirmed photoemission results, only a moderate increase of $m^{*} / m_{\mathrm{b}}$ from 2.9 to 3.1 was observed when going from $\mathrm{SrVO}_{3}$ to $\mathrm{CaVO}_{3}$, as determined from magnetic susceptibility measurement, ${ }^{[143]}$ which suggests minute changes in the carrier renormalization $Z_{k}$ through enhancement of the electron correlation. A more detailed photoemission study using a tunable UV laser source in vacuum where samples were prepared by fracturing in situ to ensure a pristine surface confirmed the suppression of spectral weight near the Fermi edge $(\sim 50 \%)$ for $\mathrm{CaVO}_{3}$ single-crystal samples compared with $\mathrm{SrVO}_{3}{ }^{[144]}$ The differences between $\mathrm{SrVO}_{3}$ and $\mathrm{CaVO}_{3}$ being relatively small inside the bulk changed when shifting from bulk to surface sensitive characterization conditions, which was attributed to the lifting of the $t_{2 g}$ orbital degeneracy on the surface by breaking the orbital degeneracy. ${ }^{[134,144]}$ Despite the considerably enhanced correlation effect on the surface, which is 
more pronounced for $\mathrm{CaVO}_{3}$, it is generally accepted that no MIT can be observed in the bulk-like form of $\mathrm{SrVO}_{3}$ and $\mathrm{CaVO}_{3}$ at all temperatures. ${ }^{[133]}$ This may stem from the fact that the distortion away from a $180^{\circ}$ bond angle of is partially compensated by a contraction in the lattice parameter, as well as the less sensitive nature of the orbital overlap of the $t_{2 g}$ orbitals compared with the $e_{g}$ orbitals for materials such as rare-earth nickelates. Other means to engineer the structure in materials accessible in epitaxial thin films, namely dimensional confinement and epitaxial strain, can be used to further enhance correlations effects in $d^{1}$ vanadates to drive these systems closer to their Mott-insulator phase.

\section{Thin-film growth of $\mathrm{AVO}_{3}$ perovskites}

As already discussed in section "MIT in binary vanadium compounds: $\mathrm{VO}_{2}$ and $\mathrm{V}_{2} \mathrm{O}_{3}$ " for the growth of binary vanadium oxides there are many techniques to deposit epitaxial thin films. Despite the challenge in binary oxides to stabilize vanadium in a particular oxidation state by ensuring the incorporation of vanadium and oxygen in a stoichiometric ratio, the growth of ternary vanadates presents additional challenges. In particular, an extra level of control is needed to achieve a $1: 1$ cation ratio, i.e., the cation occupying the $A$-site to $\mathrm{V}$ at the $B$-site. Employing target ablation techniques, such as PLD, ${ }^{[145-152]}$ sputtering, ion beam and (pulsed) electron beam evaporation ${ }^{[153-155]}$ this problem is approached by using cation stoichiometric targets and adjusting oxygen partial pressure and substrate temperature during deposition. While the film's composition is mainly set by the target's composition, achieving stoichiometric transfer from the target to the substrate has been proved difficult. The high-energy bombardment of species ablated from the target impinging on the film with large kinetic energies can cause resputtering of more volatile components or cause point defect formation from high-energy impacts of ablated species. In fact, the majority of $A \mathrm{VO}_{3}$ films studied in the literature have been grown by PLD. Although film deposition can be controlled down to the monolayer (ML) level, therefore enabling studies of dimensional controlled MITs, thicker $A \mathrm{VO}_{3}$ films were found to have low residual resistivity ratios $(R R R)$ of around $\sim 2,{ }^{[145,148,156]}$ indicating that electron transport is limited by defect scattering, being of the same order of magnitude than phonon scattering at room temperature. Therefore disorder effects are dominant, preventing to study the intrinsic electronic properties of $A \mathrm{VO}_{3}$.

In contrast, molecular beam epitaxy uses low-energy thermal evaporation to supply individual elements with low incident energies limited to thermal agitation when evaporated from the effusion cell. They are typically of the order of 0.1 $\mathrm{eV}$ therefore limiting the formation of unintentional defects in the film upon impact on the growth front. However, unlike in the case of MBE growth of compound semiconductors, such as in the archetypical case of GaAs, where the stoichiometry is self-regulated due to the volatility of $\mathrm{As},{ }^{[157]} \mathrm{AVO}_{3}$ compounds do not contain a volatile component and cannot be grown in a self-regulated manner. Therefore film stoichiometry is limited by the precision of flux calibration and the stability of fluxes during film growth, typically about $1 \%$. Recently, the hybrid MBE (hMBE) growth scheme ${ }^{[158,159]}$ has been expanded to vanadates by employing the metal-organic precursor vanadium oxytriisopropoxide (VTIP) to overcome this challenge. Here, the elements occupying the $A$-site effused from conventional thermal evaporation sources are supplied together with a flux of highly volatile VTIP molecule, which are directed at the substrate using a gas injector. The low defect density achieved in $\mathrm{SrVO}_{3}$ films grown of hMBE films have resulted in $R R R$ values larger than 220 , with a low temperature resistivity $\sim 0.1 \mu \Omega \mathrm{cm}$, and a room temperature resistivity of $\sim 20 \mu \Omega \mathrm{cm} .{ }^{[160]}$ An adsorption controlled growth window was accessed for $\mathrm{SrVO}_{3},{ }^{[161]} \mathrm{CaVO}_{3},{ }^{[162]}$ and $\mathrm{LaVO}_{3},{ }^{[163,164]}$ and recently even for the quaternary compound $\mathrm{La}_{1-x} \mathrm{Sr}_{x} \mathrm{VO}_{3}$, ${ }^{[165]}$ in which defect concentrations were found minimal and physical properties were independent of growth conditions, offering the possibility of reliable, and scalable growth.

\section{A route to enhanced correlation effects: thickness-driven MIT}

Driving a thin film from a correlated metal into insulating phases is of great interest for novel device functionalities that are based on electronic phase transition. Even though electron correlation strength existing in bulk $\mathrm{SrVO}_{3}$ and $\mathrm{CaVO}_{3}$ are not sufficient to induce an MIT, lowering the dimensionality breaks the orbital degeneracy and can enhance the correlations strength, which may be sufficient to trigger an MIT. ${ }^{[154,155,166,167]}$ Normally, three $\mathrm{V} 3 d t_{2 g}$ orbitals $\left(d_{x y}, d_{x z}, d_{y z}\right)$ are degenerate in the bulk case. As thickness is reduced, bonding normal to the surface is interrupted and orbitals with projection normal to the surface shift to higher energy and become gradually depopulated. This orbital selective quantum confinement is unique to TMOs and density functional theory + dynamical mean-field theory calculation have shown that even though two layers of $\mathrm{SrVO}_{3}$ on $\mathrm{SrTiO}_{3}$ substrate were insulating, they are at the very verge of an MIT and sensitive to external parameters, such as pressure, temperature or electrical field. ${ }^{[168]}$

Motivated by these predictions Yoshimatsu et al. probed ultrathin $\mathrm{SrVO}_{3}$ films grown on $\mathrm{Nb}: \mathrm{SrTiO}_{3}$ and provided the spectroscopic evidence for a thickness-driven MIT. ${ }^{[167]}$ Figure 13(a) shows the evolution of the DOS measured from photoemission spectra as a function of $\mathrm{SrVO}_{3}$ thickness in unit cells. The spectra have been symmetrized with respect to $E_{\mathrm{F}}$, which enables tracking the energy gap as the thickness is reduced. In the ultrathin limit, i.e., a film thickness between 1 and $5 \mathrm{ML}$ a pseudogap (PG) was present, the DOS at the Fermi level was suppressed and transferred into the incoherent side peak that emerged at a binding energy of $\sim 1.6 \mathrm{eV}$. The spectral weight transfer with decreasing thickness along with the energy gap formation was interpreted as resulting of an increased correlation strength. These spectroscopic data suggested that a Mott-insulator phase emerged out of the correlated metal phase in the ultrathin limit. The trends in the position of 



Figure 13. (a) Photoemission spectra from $\mathrm{SrVO}_{3}$ thin films with thickness indicated in $\mathrm{ML}$. (b) Intensity (blue circles) and position (green triangles) of the coherent quasi-particle peak and the incoherent peak (red squares) relative to the Fermi level $E_{F}$, extracted from the photoemission data shown in (a), revealing a transition from the $\mathrm{SrVO}_{3}$ metal phase at a large layer thickness (>8 ML) to a gapped insulating phase (MI) in the ultrathin limit between 3 and $1 \mathrm{ML}$. (c) Temperature-dependent conductivity of $\mathrm{SrTiO}_{3}-\mathrm{SrVO}_{3}-\mathrm{SrTiO}_{3}$ heterostructures with varying $\mathrm{SrVO}_{3}$ layer thickness. (d) Free-carrier density $n$ of samples measured in (c) as a function of $\mathrm{SrVO}_{3}$ layer thickness with a two orders of magnitude reduction for the 3-ML-thick $\mathrm{SrVO}_{3}$, evidence for a pronounced carrier localization effect in the ultrathin limit of $\mathrm{SrVO}_{3}$ [panel (a) and (b) are reprinted from Ref. 167. Copyrighted by the American Physical Society].

coherent quasi-particle and incoherent side peak as well as intensity of the coherent quasi-particle intensity with thickness are summarized in Fig. 13(b). While the position of the side peaks remained unaltered with thickness, suggesting a constant Coulomb repulsion energy $U$, the coherent quasi-particle peak shifts away from the Fermi level in the PG region along with an intensity reduction. ${ }^{[167]}$ These spectral features have been confirmed by transport measurements performed on $\mathrm{SrTiO}_{3}-$ $\mathrm{SrVO}_{3}-\mathrm{SrTiO}_{3}$ structures grown on (001) $\left(\mathrm{La}_{0.3} \mathrm{Sr}_{0.7}\right)$ $\left(\mathrm{Al}_{0.65} \mathrm{Ta}_{0.35}\right) \mathrm{O}_{3}$ by hybrid MBE; see Figs. 13(c) and 13(d). Here the 15-nm-thick $\mathrm{SrTiO}_{3}$ buffer and cap layer were kept constant, which imposed the dimensional confinement over the $\mathrm{SrVO}_{3}$ layer with thicknesses of a few perovskite unit cells. For large $\mathrm{SrVO}_{3}$ thicknesses the conductivity monotonically decreased with increasing temperature, which is characteristic of metallic conduction. While for a $\mathrm{SrVO}_{3}$ thickness of $8 \mathrm{ML}$ the metallic behavior was still dominant, despite the fact the electrical conductivity was reduced due to an enhanced surface scattering effect in the ultrathin limit. The transport characteristics of the 3-ML-thick $\mathrm{SrVO}_{3}$ film was dramatically different. Here, the temperature dependent conductivity had a positive slope indicating the formation of a fully gapped insulating phase. Carrier concentration extracted from Hall effect measurements are shown in Fig. 13(d) and revealed a 100× reduction in the bulk carrier density of the 3-ML-thick $\mathrm{SrVO}_{3}$ layer compared with the samples with metallic conduction. The orders-of-magnitude reduction in the number of delocalized carriers is temperature independent, consistent with the picture of a complete $D O S$ transfer away from $E_{\mathrm{F}}$. The large nominal sheet carrier concentration of $\sim 2 \times 10^{15} \mathrm{~cm}^{-2}$, expected for a 3-ML-thick $\mathrm{SrVO}_{3}$, would require a similar defect concentration to trap nearly all delocalized carriers, an 
unlikely scenario given the perfection of the material demonstrated by hybrid MBE, ${ }^{[160,169]}$ thus precluding disorder-related extrinsic effects and supporting the spectroscopic results. ${ }^{[170]}$

\section{Correlated metals as TCs}

Although bulk $\mathrm{SrVO}_{3}$ and $\mathrm{CaVO}_{3}$ are far from the Mott transition and the Mott gap can only be opened in the ultrathin limit, the strong electron-electron correlation alters the electrical and optical properties in ways that makes them interesting for the application as thin-film TC materials. ${ }^{[133]}$ TC materials are a key component for virtually any modern electro-optical devices, from flat-panel screens in smart phones and televisions, to photovoltaic devices. The properties of high optical transparency and high electrical conductivity are mutually exclusive - the large amount of free carriers required to effectively conduct electricity will also cause to reflect the visible light, which presents a distinct challenge at a fundamental materials design level.

The two effects relevant to optical transmission are reflection at low energies, caused by the free carrier response to light, and absorption at higher energies due to interband transitions. Minimizing these effects are the cornerstones for the design strategy of TCs. This has been achieved in the past by choosing a wide band gap semiconductors to avoid interband transitions in the visible spectrum, and to degenerately dope it to make it sufficiently conducting. While the low-energy cutoff for high transmission is determined by the reflection edge due to the free carrier response, quantified by the screened plasma frequency, a sufficiently large band gap ensured that interband transitions occurred at photon energies above the visible spectrum, opening a transmission window over the entire visible range of photon energies from 1.75 to $3.25 \mathrm{eV}$. The trade-off between the mutually exclusive properties of optical transparency and electrical conductivity can be best seen by comparing the screened plasma frequency $\omega_{\mathrm{p}}=\left(q / \sqrt{\varepsilon_{0} \varepsilon_{\mathrm{r}}}\right) \sqrt{n / m *}$ where $q$ is the electron charge, $\varepsilon_{0}$ and $\varepsilon_{\mathrm{r}}$ are the vacuum and relative permittivity, respectively, $n$ is the charge carrier concentration, and $m^{*}$ is the effective carrier mass, to the conductivity $\sigma=q^{2} \tau_{\mathrm{s}}\left(n / m^{*}\right)$ where $\tau_{\mathrm{s}}$ is the mean scattering time of the free carriers. Doping the semiconductor increases $n$, which increases $\sigma$ and simultaneously pushes the reduced plasma energy $E_{\mathrm{p}}=\hbar \omega_{\mathrm{p}}$ toward the onset of the visible spectrum. For the current work-horse material, Sn-doped indium oxide (ITO), electrical conductivities up to $\sim 1 \times 10^{4} \mathrm{~S} / \mathrm{cm}$ were achieved with $E_{\mathrm{p}} \approx 0.77 \mathrm{eV}$. This is well below the low-energy onset of the visible spectrum thus leaving room to further increase conductivity, which was not possible because the maximum carrier concentration of $\sim 3 \times 10^{21} / \mathrm{cm}^{3}$ in ITO marks the solubility limit of dopants. In addition, pronounced charged impurity scattering from the dopant atoms occurred at these dopant concentrations, detrimentally affecting the room temperature carrier mobility reducing the electrical conductivity. ${ }^{[171]}$ For the application as a TC both phenomena mark the intrinsic limits of degenerately doped wide band semiconductors in general, and ITO in particular.

This fundamental limit can be overcome when focusing the attention to materials with a sizeable electron correlation. The effective carrier mass of materials with negligible electronelectron interaction, such as conventional semiconductors with bands derived from $s$ - and $p$-orbitals, is solely determined by the band effective mass. In contrast, materials exhibiting strong electron-electron correlation provide an additional design DOF: modulation of carrier effective mass $m^{*}$ through electron correlation. Therefore, $\omega_{\mathrm{p}}$ and $\sigma$ can not only be modified by changing the carrier concentration $n$, as in the case of conventional semiconductors, but also by engineering the correlation strength. In particular, materials with a metal-like highcarrier concentration and strong correlation resulting in a renormalized and thus heavier electron effective mass can meet the requirements of a sufficiently small $\omega_{\mathrm{p}}$ despite a large $\sigma$. The key factor to balance conductivity and plasma frequency is $n$ / $m^{*}$, but rather than finding semiconductors with a small effective mass $m^{*}$ to compensate for the limited $n$, in case of correlated metals the high-carrier concentration $n$ is compensated by a large carrier effective mass $m^{*}$. While the high carrier concentration renders normal metals, such as aluminum, copper, gold, and silver unsuitable as TCs, exhibiting reduced plasma frequencies within or even beyond the visible range, the reduced plasma energy for a correlated metal with large carrier effective mass can be below the visible range, yet higher than the plasma energy of ITO. As shown in Fig. 14(a), correlated metals $\mathrm{SrVO}_{3}$ and $\mathrm{CaVO}_{3}$ with a carrier concentration of $\sim 2 \times 10^{22} / \mathrm{cm}^{3}$ have proven this hypothesis with an experimentally determined screened plasma frequencies as low as 1.33 and $1.29 \mathrm{eV}$, well below the critical value of $1.75 \mathrm{eV}$ and larger than $E_{\mathrm{p}}$ of ITO of $0.77 \mathrm{eV}$. A one order of magnitude higher electrical conductivity of $\mathrm{SrVO}_{3}$ and $\mathrm{CaVO}_{3}$ compared with ITO are testaments of the tremendous potential of correlated metals to push the carrier reflection edge closer to the $1.75 \mathrm{eV}$.

For a material to be a promising TC material, a low optical absorption is mandatory. The band structure of the vanadates are favorable because the $\mathrm{V} 3 d$ band is energetically isolated from the higher lying V $3 d e_{g}$ bands and the lower lying valence bands derived from $\mathrm{O} 2 p$. In case of $\mathrm{SrVO}_{3}$ a sizeable interband transition from $\mathrm{O} 2 p$ to $\mathrm{V} 3 d t_{2 g}$ near the UV edge is present, while other interband transitions within the visible spectrum are largely suppressed, resulting in a compromised optical transmissivity compared with ITO. Nevertheless, the combination of very high electrical conductivities and acceptable optical transmission in the visible spectrum makes vanadates ideal TC candidates with competitive figure of merits (FOMs) on par with ITO, as shown in Fig. 15(a).

The film thickness scaling is another important aspect of a TC material in real-world applications: a decrease of electrical conductivity due to an enhanced film surface scattering occurs when film thickness approaches electron mean free path (EMFP) $\Gamma=\hbar\left(3 \pi^{2}\right)^{1 / 3}\left(\tau_{\mathrm{s}} / n^{2 / 3}\right)\left(n / m^{*}\right)$. While thinner films result 



Figure 14. (a) Optimization of electrical conductivity $\sigma$ of correlated metals while keeping reflection edge, represented by the screened plasma frequency $\omega_{\mathrm{p}}$, below the visible spectrum, indicated by the rainbow. (b) The electrical conductivity as a function of film thickness of Ag, Au, polycrystalline IT0, pulsed laser deposition (PLD)-grown ITO and epi-ITO, $\mathrm{SrVO}_{3}$, and $\mathrm{CaVO}_{3}$ (adapted from Ref. 133).

(a)
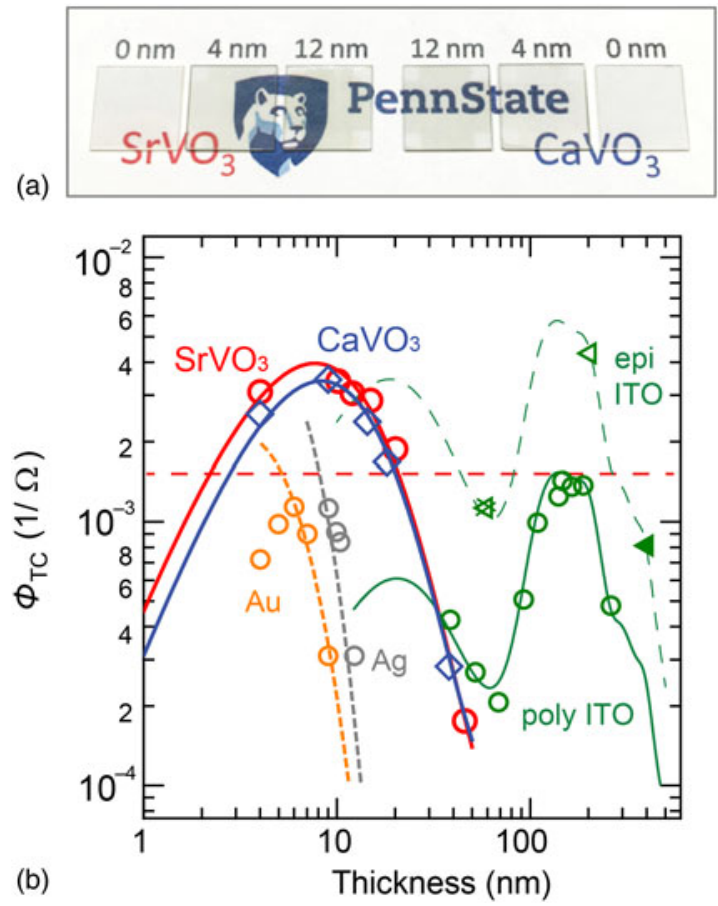

Figure 15. (a) Photographs from left to right are a bare $\left(\mathrm{La}_{0.3} \mathrm{Sr}_{0.7}\right)$ $\left(\mathrm{Al}_{0.65} \mathrm{Ta}_{0.35}\right) \mathrm{O}_{3}$ (LSAT) substrate, $4 \mathrm{~nm} \mathrm{SrVO}{ }_{3}$ on LSAT, $12 \mathrm{~nm} \mathrm{SrVO}{ }_{3}$ on LSAT, $12 \mathrm{~nm} \mathrm{CaVO}{ }_{3}$ on $\mathrm{SrLaAlO}_{4}$ (SLAO) substrate, $4 \mathrm{~nm} \mathrm{CaVO}{ }_{3}$ on LSAO and a bare LSAO substrates on colored background, respectively. (b) FOM $\Phi_{\mathrm{TC}}=T^{10} / R_{\mathrm{S}}$ for free-standing transparent conducting film as a function of thickness with average transmittance in the visible spectrum and sheet resistance $R_{S}$ (adapted from Ref. 133). in a higher optical transparency it in turn reduces electrical conductivity. The EMFP of $\mathrm{SrVO}_{3}\left(\Gamma_{\mathrm{SvO}} \approx 5.6 \mathrm{~nm}\right)$ and $\mathrm{CaVO}_{3}$ $\left(\Gamma_{\mathrm{CVO}} \approx 3.9 \mathrm{~nm}\right)$ were found much shorter compared with conventional metals $\left(\Gamma_{\mathrm{Au}} \approx 50 \mathrm{~nm}\right.$ for gold, and $\Gamma_{\mathrm{Ag}} \approx 52 \mathrm{~nm}$ for silver) or $\mathrm{ITO}^{[172]}\left(\Gamma_{\text {ITO }} \approx 17 \mathrm{~nm}\right.$ for polycrystalline film). The favorable, while shorter, EMFP in correlated metals is a direct consequence of the larger effective mass and higher carrier concentration since $\Gamma \sim n^{1 / 3} / m^{*}$. Technologically, this shorter EMFP is beneficial, allowing not only thinner films, but also reduces the sensitivity to grain boundary scattering, making the electrical conductivity more tolerant to the film's microstructural effects. The thickness dependence of a material as TC can be quantified by a FOM, which is taken to be $\Phi_{\mathrm{TC}}=$ $T^{10} / R_{\mathrm{S}}$, where $R_{\mathrm{S}}$ is the sheet resistance and $T$ is the transmission coefficient. ${ }^{[133]}$ As shown in Fig. 15(b), $\mathrm{SrVO}_{3}$ and $\mathrm{CaVO}_{3}$ have a comparable FOM a maximum value $\Phi_{\mathrm{TC}} \approx$ $4 \times 10^{-3} 1 / \Omega$ for 10 -nm-thick films striking the best balance between the mutually excluding properties of electrical conductivity and optical transparency. For comparison the maximum FOM for epi-ITO of $\Phi_{\mathrm{TC}} \approx 6 \times 10^{-3} 1 / \Omega$ is on par, but achieved at a much larger film thickness of above $100 \mathrm{~nm}$. This comparison exemplifies the economic advantage of correlated metals as TCs that can be achieved through faster manufacturing time and being composed out of earth abundant, less expensive starting materials. This holds true in particular for the main constituent of ITO, namely indium, being more than 30 times more expensive than the elements $\mathrm{Sr}$ and $\mathrm{V}$. The much reduced film thickness and lower costs of the starting material provide a tremendous driving force toward overcoming the materials synthesis challenge to scaling up the growth of vanadate thin 
films toward high-throughput, low-temperature deposition processes needed to commercialize vanadates as novel transparent electrode material.

\section{The Mott-insulating phase in rare-earth vanadates}

The electronic configuration of rare-earth vanadates is different to alkaline earth vanadates, the trivalent rare-earth ions occupying the $A$-site leave vanadium in a $3^{+}$state resulting in a $d^{2}$ configuration. All rare-earth compounds exhibit orthorhombic distortion (space group Pbnm). ${ }^{[11]}$ Despite the increase of the ionic radius of vanadium by about $20 \%$ being in a lower valence state; all rare-earth ions have a much larger radius. The carriers are localized, the $d_{x y}$ orbital is lowered in energy compared with the two other $t_{2 g}$ orbitals $d_{y z}$ and $d_{z x}$ and a sizeable Mott gap is formed. ${ }^{[173]}$ At low temperatures spin and orbital ordering effects occur that are coupled to the degree of orthorhombic distortion and thus the ionic radius of the $A$-site cation, as shown in Fig. 16. In moving from smaller $(\mathrm{Yb})$ toward larger (La) cations, antiferromagnetic ordering of orbitals emerge $\sim 125 \mathrm{~K}$ with spins aligned in the G-type configuration $(\pi, \pi, \pi)$ (indicating out of phase, hence $\pi$, along the $x$, $y$, and $z) .{ }^{[174]}$ The spins reorder in the C-type antiferromagnetic fashion $(\pi, \pi, 0)$ below $\sim 100 \mathrm{~K}$, and finally below $80 \mathrm{~K}$, orbital ordering switches to C-type as well. As the radius of the rare-earth ion is increased, thus increasing the bonding angle and reducing the orthorhombic distortion toward the cubic perovskite, the type of spin and orbital ordering and transition temperatures change accordingly. For the vanadate with the largest rare-earth ion occupying the $A$-site of the perovskite structure, $\mathrm{LaVO}_{3}$, the orbital and spin ordering occur at nearly the same temperature with spin order emerging first, followed by the orbital order. These ordering as well as the slight ferromagnetic canting can be understood in the context of the Goodenough-Kanamori rules, ${ }^{[175,176]}$ along with the evolution of the orthorhombic-to-monoclinic distortion dependent on the change in tolerance factor with increasing cation radius.

While in the case of rare-earth vanadates the underlying physics and the complex interplay of spin and orbital ordering is rich, the aspect of application of these films has so far been pursued to a lesser degree. Interestingly $\mathrm{LaVO}_{3}$ is well suited for photovoltaic applications, exhibiting the largest Mott gap of $\sim 1.1 \mathrm{eV}$ in the $R \mathrm{VO}_{3}$ series formed between the $d_{x y}$ and $d_{y z} / d_{z x}$ bands. ${ }^{[177]}$ The transition is direct and in the optimum range of $1-1.5 \mathrm{eV}$ for energy harvesting from the solar spectrum. Assmann et al. have proposed to utilize $\mathrm{LaVO}_{3} / \mathrm{SrTiO}_{3}$ oxide heterostructures for high-efficiency photovoltaic cells. ${ }^{[177]}$ While in $\mathrm{SrTiO}_{3} \mathrm{Sr}$ and $\mathrm{Ti}$ have nominal valence states of $2^{+}$and $4^{+}$, and therefore alternating $\mathrm{Sr}^{2+} \mathrm{O}^{2-}$ and $\mathrm{Ti}^{4+} \mathrm{O}_{2}^{4-}$ planes are charge neutral, in $\mathrm{LaVO}_{3}$ both $\mathrm{La}$ and $\mathrm{V}$ have nominal valence of $3^{+}$, rendering the $\mathrm{LaO}$ and $\mathrm{VO}_{2}$ surfaces polar with alternating charge +1 and -1 , respectively. The polar discontinuity formed at the $\mathrm{LaVO}_{3} / \mathrm{SrTiO}_{3}$ interface can be compensated by electrons transferred from the surface of $\mathrm{LaVO}_{3}$ to the interface. Similar to the well-studied example $\mathrm{LaAlO}_{3} / \mathrm{SrTiO}_{3}^{[39]}$ this will set up an

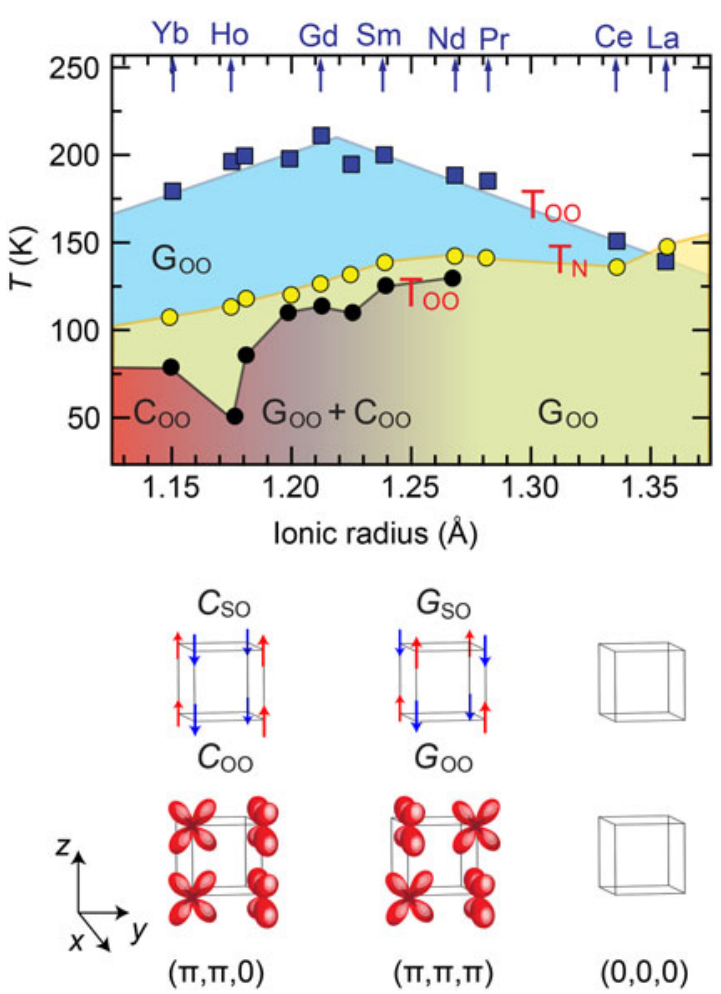

Figure 16. Temperature dependence of orbital ordering (subscript 00 ) and spin ordering (subscript $\mathrm{N}$ ) versus ionic radius for the rare-earth vanadates $\left(R V O_{3}\right) . G$ and $C$ indicated the two different ordering types schematically shown in the bottom panel (adapted with permission from Ref. 174. Copyrighted by the American Physical Society).

electrical field across $\mathrm{LaVO}_{3}{ }^{[149]}$ The large absorption coefficient of $\mathrm{LaVO}_{3}$ causes effective electron-hole pair formation, which are separated by the imposed electric field and collected on the bottom electrode, the 2D electron gas formed at the $\mathrm{LaVO}_{3} / \mathrm{SrTiO}_{3}$ interface and a transparent electrode deposited on $\mathrm{LaVO}_{3}$. The Mott gap of $\mathrm{LaVO}_{3}$ is direct so that incident photons can effectively create electron-hole pairs. The high extraction efficiency due to the small distance to the electrodes makes this all-oxide solar cell design attractive. Wang et al. ${ }^{[178]}$ also pointed out that both, $d-d$ transition between LHB and UHB and $p-d$ transition from $\mathrm{O} 2 p$ into the $\mathrm{V} 3 d$, give rise to light absorption in the visible and UV spectrum. Density functional theory has predicted that the absorption coefficient of $\mathrm{LaVO}_{3}$ is even higher than that of $\mathrm{CdTe},{ }^{[177]}$ a conventional semiconductor dominating thin-film solar cells on the market. ${ }^{[179]}$ The ability to epitaxially integrate structurally compatible, closely lattice-matched perovskite material with good TCs and absorbers with different band gaps, such as $\mathrm{LaFeO}_{3},{ }^{[180]}$ could further boost the efficiency of these all-oxide multi-junction solar cells beyond commonly accepted limits.

\section{Conclusion and outlook}

In the current era of information technology, electronic and photonic devices are becoming more complex, while 
multifunctional, demanding progress in materials science to develop integration strategies and to find new ways to augment and improve current devices that transmit and receive, process, and store data. While the transition from personal to mobile computing was not only driven by advances in transistor and memory technology, also other key elements, such as battery and wireless communication, touch display panels, and MEMS sensor technology have become critical too. The dramatic diversification of the components opens up a large materials space that expands beyond conventional semiconductor materials.

A rapid change of the type and complexity of computation problems has been witnessed in the recent past. The growth in the number of data sets and their size has shifted the focus away from small data precision computing to big-data approximate computing, efficient sorting algorithms, and encryption routines for secure data transfer. A breakthrough to realize augmented reality requires energy-efficient pattern recognition and processing capabilities. In anticipation of these paradigm shifts, computing that utilizes CMOS technology and Boolean logic, may not always offer the best solution for such diverse, and newly defined problem sets. Non-classical computing schemes known from the early days of electronic computing may be revived and with it the demand for other hardware solutions made from different materials.

These emerging trends in computation technology will trigger a renaissance in the science and engineering of electronic materials. The discovery of new materials, their implementation into mature material platforms will enable the construction of faster and more energy-efficient hardware solutions. The level of CMOS technology has been relentlessly pushed toward the intrinsic limits. The technological effort is overwhelming, but performance advancements are too small to justify the increasing the fabrication costs. This favors the strategy to look for new material opportunities. These current trends have propelled decades-old material curiosities to the forefront of technological applications. In particular, bringing strongly correlated electronic PCMs of TMOs into real consideration for device applications is a guarantor to find new opportunities and advancing this field.

Here we have reviewed the status of the field of the vanadium-based oxide materials, and outlined areas for future research endeavors. We discussed how the MITs in the binary $\mathrm{VO}_{2}$ phases can be used to augment current devices, in particular, field-effect transistors, and can be used as coupled oscillator circuit elements to enable non-Boolean computing, and as a solid-state RF switches. Moving on to the chemically more complex ternary perovskite vanadates, we discussed electron correlation effects in the metals $\mathrm{CaVO}_{3}$ and $\mathrm{SrVO}_{3}$, which can be used as highly conductive electrode that is compatible within complex all-perovskite epi-stacks. We have emphasized that even far away from an MIT strong electron correlation effects offer new ways to balance the antagonistic properties of high electrical conductivity and optical transparency, which break ground for a new design principle to develop cheaper and better materials for the ubiquitous application of TCs beyond the realms of degenerately doped wide band gap semiconductors. Finally, we discussed the rare-earth perovskite vanadates, where strong correlations pushed the ground state to a gapped Mott-insulating phase with a complex interplay between orbital and spin order. $\mathrm{LaVO}_{3}$, in particular, with its correlation-induced $\sim 1.1 \mathrm{eV}$ direct band gap, has potential to exploit the properties of oxides to realize a novel, all-oxide multi-junction solar cell. In closing, although research into the vanadate-based oxides has spanned the better part of a century, there are many opportunities in future endeavors to further improve our understanding of these novel phases, to further perfect the thin-film synthesis and ultimately to put it to use in practical applications to benefit our society.

\section{Acknowledgments}

M. B. and R. E. H. acknowledge the Department of Energy (Grant DE-SC0012375) for support during preparation of the manuscript. R. E. H., D. G. S., S. D., L. Z., H. P., and N. S. acknowledge support through the Office of Naval Research (ONR) through Award N00014-11-1-0665. S. D. and N. S. also acknowledge the Center for Low Energy Systems Technology (LEAST), one of six centers of STARnet, a Semiconductor Research Corporation (SRC) program sponsored by MARCO and DARPA, and Intel Customized SRC program. Support from the National Science Foundation, the MRSEC Center for Nanoscale Science at Penn State through Grant no. DMR1420620 for J. L. and H. Z., and through Grant no. DMR1352502 for L. Z. and R. E. H. is acknowledged. We would like to thank Lindsay Noskin for help with preparation of Fig. 4.

\section{References}

1. G.E. Moore: Cramming more components onto integrated circuits. Electronics 38, 114-117 (1965).

2. J. Koomey and S. Naffziger: Moore's law might be slowing down, but not energy efficiency. IEEE Spectrosc. (2015). www.spectrum.ieee.org/ computing/hardware/moores-law-might-be-slowing-down-but-notenergy-efficiency (accessed February 1, 2017).

3. T. Cross: Double, double, toil and trouble. Econ. Mar. (2016). www. economist.com/technology-quarterly/2016-03-12/after-moores-law (accessed February 1, 2017).

4. P.A. Lee and X-G. Wen: Doping a Mott insulator: physics of hightemperature superconductivity. Rev. Mod. Phys. 78, 17-85 (2006).

5. Y. Tokura and Y. Tomioka: Colossal magnetoresistive manganites. J. Magn. Magn. Mater. 200, 1-23 (1999).

6. A. Schilling, M. Cantoni, J.D. Guo, and H.R. Ott: Superconductivity above $130 \mathrm{~K}$ in the $\mathrm{Hg}-\mathrm{Ba}-\mathrm{Ca}-\mathrm{Cu}-0$ system. Nature 363, 56-58 (1993).

7. J. Heber: Enter the oxides. Nature 459, 28 (2009).

8. Y. Tokura: Correlated-electron physics in transition-metal oxides. Phys. Today 56, 50-55 (2003).

9. Y. Tokura: Orbital physics in transition-metal oxides. Science $\mathbf{2 8 8}, \mathbf{4 6 2 -}$ 468 (2000).

10. M. Imada, A. Fujimori, and Y. Tokura: Metal-insulator transitions. Rev. Mod. Phys. 70, 1039-1263 (1998).

11. J.B. Goodenough: Electronic and ionic transport properties and other physical aspects of perovskites. Rep. Prog. Phys. 67, 1915-1993 (2004).

12. J.B. Goodenough: Anomalous properties of the vanadium oxides. Annu. Rev. Mater. Sci. 1, 101-138 (1971). 
13. T. Yamauchi, M. Isobe, and Y. Ueda: Charge order and superconductivity in vanadium oxides. Solid State Sci. 7, 874-881 (2005).

14. N.W. Ashcroft and N.D. Mermin: Solid State Physics (Rinehart, and Winston, Holt, 1976).

15. M. Cardona and F.H. Pollak: Energy-band structure of germanium and silicon: the k.p method. Phys. Rev. 142, 530-543 (1966).

16. A. Fujimori, T. Yoshida, K. Okazaki, T. Tsujioka, K. Kobayashi, T. Mizokawa, M. Onoda, T. Katsufuji, Y. Taguchi, and Y. Tokura: Electronic structure of Mott-Hubbard-type transition-metal oxides. J. Electron Spectros. Relat. Phenom. 117, 277-286 (2001).

17. N.F. Mott and L. Friedman: Metal-insulator transitions in $\mathrm{VO}_{2}, \mathrm{Ti}_{2} \mathrm{O}_{3}$ and $\mathrm{Ti}_{2-\mathrm{x}} \mathrm{V}_{\mathrm{x}} \mathrm{O}_{3}$. Philos. Mag. 30, 389-402 (1974).

18. J. Zaanen, G.A. Sawatzky, and J.W. Allen: Band gaps and electronic structure of transition-metal compounds. Phys. Rev. Lett. 55, 418421 (1985)

19. L. Pauling: The principles determining the structure of complex ionic crystals. J. Am. Chem. Soc. 51, 1010-1026 (1929).

20. F.J. Morin: Oxides which show a metal-to-insulator transition at the Neel temperature. Phys. Rev. Lett. 3, 34-36 (1959).

21. J. Feinleib and W. Paul: Semiconductor-to-metal transition in $\mathrm{V}_{2} \mathrm{O}_{3}$. Phys. Rev. 155, 841-850 (1967).

22. L.A. Ladd and W. Paul: Optical and transport properties of high quality crystals of $\mathrm{V}_{2} \mathrm{O}_{4}$ near the metallic transition temperature. Solid State Commun. 7, 425-428 (1969).

23. P. Hansmann, A. Toschi, G. Sangiovanni, T. Saha-Dasgupta, S. Lupi, M. Marsi, and K. Held: Mott-Hubbard transition in $\mathrm{V}_{2} \mathrm{O}_{3}$ revisited. Phys. Status Solidi 250, 1251-1264 (2013).

24. M.M. Qazilbash, A.A. Schafgans, K.S. Burch, S.J. Yun, B.G. Chae, B.J. Kim, H.T. Kim, and D.N. Basov: Electrodynamics of the vanadium oxides $\mathrm{VO}_{2}$ and $\mathrm{V}_{2} \mathrm{O}_{3}$. Phys. Rev. B 77, 075124 (2008)

25. P.D. Dernier and M. Marezio: Crystal structure of the low-temperature antiferromagnetic phase of $\mathrm{V}_{2} \mathrm{O}_{3}$. Phys. Rev. B 2, 3771-3776 (1970).

26. I. Chatterjee: Interplay of orbital ordering and exchange interaction in $\mathrm{V}_{2} \mathrm{O}_{3}$. J. Phys. Condens. Matter 13, 109-114 (2001).

27. J.S. Griffith: The Theory of Transition-Metal Ions (Cambridge University Press, Cambridge, 1961).

28. R. Shiina, F. Mila, F-C. Zhang, and T.M. Rice: Atomic spin, molecular orbitals, and anomalous antiferromagnetism in insulating $\mathrm{V}_{2} \mathrm{O}_{3}$. Phys. Rev. B 63, 144422 (2001)

29. A. Tanaka: Electronic structure and phase transition in $\mathrm{V}_{2} \mathrm{O}_{3}$ : importance of $3 \mathrm{~d}$ spin-orbit interaction and lattice distortion. J. Phys. Soc. Japan 71, 1091-1107 (2002).

30. F.A. Chudnovskii, E.I. Terukov, and D.I. Khomskii: Insulator-metal transition in $\mathrm{V}_{3} \mathrm{O}_{5}$. Solid State Commun. 25, 573-577 (1978).

31. N.N. Khoi, T.R. Simon, and H.K. Eastwood: Phase transition in $\mathrm{V}_{3} \mathrm{O}_{5}$. Mater. Res. Bull. 11, 873-878 (1976).

32. N. Bahlawane and D. Lenoble: Vanadium oxide compounds: structure, properties, and growth from the gas phase. Chem. Vap. Depos. 20, 299-311 (2014)

33. D.B. McWhan and T.M. Rice: Critical pressure for the metalsemiconductor transition in $\mathrm{V}_{2} \mathrm{O}_{3}$. Phys. Rev. Lett. 22, 887-890 (1969).

34. J. Cao, Y. Gu, W. Fan, L.Q. Chen, D.F. Ogletree, K. Chen, N. Tamura, M. Kunz, C. Barrett, J. Seidel, and J. Wu: Extended mapping and exploration of the vanadium dioxide stress-temperature phase diagram. Nano Lett. 10, 2667-2673 (2010).

35. Y. Gu, J. Cao, J. Wu, and L-Q. Chen: Thermodynamics of strained vanadium dioxide single crystals. J. Appl. Phys. 108, 083517 (2010).

36. S. Yonezawa, Y. Muraoka, Y. Ueda, and Z. Hiroi: Epitaxial strain effects on the metal-insulator transition in $\mathrm{V}_{2} \mathrm{O}_{3}$ thin films. Solid State Commun. 129, 245-248 (2004).

37. Y. Muraoka and Z. Hiroi: Metal-insulator transition of $\mathrm{VO}_{2}$ thin films grown on $\mathrm{TiO}_{2}$ (001) and (110) substrates. Appl. Phys. Lett. 80, 583 (2002).

38. C.H. Ahn, A. Bhattacharya, M. Di Ventra, J.N. Eckstein, C.D. Frisbie, M.E. Gershenson, A.M. Goldman, I.H. Inoue, J. Mannhart, A.J. Millis, A.F. Morpurgo, D. Natelson, and J.-M. Triscone: Electrostatic modification of novel materials. Rev. Mod. Phys. 78, 1185-1212 (2006).

39. A. Ohtomo and H.Y. Hwang: A high-mobility electron gas at the $\mathrm{LaAlO}_{3} /$ $\mathrm{SrTiO}_{3}$ heterointerface. Nature 427, 423-426 (2004).
40. A.M. Goldman: Electrostatic gating of ultrathin films. Annu. Rev. Mater. Res. 44, 45-63 (2014).

41. M. Nakano, K. Shibuya, D. Okuyama, T. Hatano, S. Ono, M. Kawasaki, Y. Iwasa, and Y. Tokura: Collective bulk carrier delocalization driven by electrostatic surface charge accumulation. Nature 487, 459-462 (2012).

42. J. Jeong, N. Aetukuri, T. Graf, T.D. Schladt, M.G. Samant, and S.S.P. Parkin: Suppression of metal-insulator transition in $\mathrm{VO}_{2}$ by electric field-induced oxygen vacancy formation. Science 339, 1402-1405 (2013).

43. H. Ji, J. Wei, and D. Natelson: Modulation of the electrical properties of $\mathrm{VO}_{2}$ nanobeams using an ionic liquid as a gating medium. Nano Lett. 12 , 2988-2992 (2012).

44. Y. Zhou and S. Ramanathan: Mott memory and neuromorphic devices. Proc. IEEE 103, 1289-1310 (2015).

45. Y-B. Kang: Critical evaluation and thermodynamic optimization of the V0-V0 ${ }_{2.5}$ system. J. Eur. Ceram. Soc. 32, 3187-3198 (2012).

46. Y. Hirotsu and $\mathrm{H}$. Sato: Periodic microsyntaxy in $\mathrm{V}_{\mathrm{n}} \mathrm{O}_{2 \mathrm{n}-1}$. Mater. Res. Bull. 15, 41-44 (1980).

47. H. Katzke, P. Tolédano, and W. Depmeier: Theory of morphotropic transformations in vanadium oxides. Phys. Rev. B 68, 024109 (2003).

48. I. Barin: Thermochemical Data of Pure Substances (VCH Verlagsgesellschaft $\mathrm{mbH}$, Weinheim (Federal Republic of Germany) and VCH Publishers, Inc., New York, NY, 1995).

49. A.Z. Moshfegh and A. Ignatiev: Formation and characterization of thin film vanadium oxides: auger electron spectroscopy, $x$-ray photoelectron spectroscopy, $x$-ray diffraction, scanning electron microscopy, and optical reflectance studies. Thin Solid Films 198, 251-268 (1991).

50. J.W. Tashman, J.H. Lee, H. Paik, J.A. Moyer, R. Misra, J.A. Mundy, T. Spila, T.A. Merz, J. Schubert, D.A. Muller, P. Schiffer, and D.G. Schlom: Epitaxial growth of $\mathrm{VO}_{2}$ by periodic annealing. Appl. Phys. Lett. 104, 063104 (2014).

51. A. Kar, N. Shukla, E. Freeman, H. Paik, H. Liu, R. Engel-Herbert, S.S.N. Bharadwaja, D.G. Schlom, and S. Datta: Intrinsic electronic switching time in ultrathin epitaxial vanadium dioxide thin film. Appl. Phys. Lett. 102, 072106 (2013)

52. H. Paik, J.A. Moyer, T. Spila, J.W. Tashman, J.A. Mundy, E. Freeman, N. Shukla, J.M. Lapano, R. Engel-Herbert, W. Zander, J. Schubert, D.A. Muller, S. Datta, P. Schiffer, and D.G. Schlom: Transport properties of ultra-thin $\mathrm{VO}_{2}$ films on (001) $\mathrm{TiO}_{2}$ grown by reactive molecular-beam epitaxy. Appl. Phys. Lett. 107, 163101 (2015).

53. L.L. Fan, S. Chen, Y.F. Wu, F.H. Chen, W.S. Chu, X. Chen, C.W. Zou, and Z.Y. Wu: Growth and phase transition characteristics of pure M-phase $\mathrm{VO}_{2}$ epitaxial film prepared by oxide molecular beam epitaxy. Appl. Phys. Lett. 103, 131914 (2013).

54. M. Della Negra, M. Sambi, and G. Granozzi: Ultrathin $\mathrm{VO}_{x} / \mathrm{TiO}_{2}(110)$ $(x \approx 1)$ film preparation by controlled oxidation of metal deposits. Surf. Sci. 436, 227-236 (1999)

55. T.A. Pak: Vapor pressure $\mathrm{V}_{2} \mathrm{O}_{5}$. Zhurnal Fiziheskoi Khimii 46, 2121 (1972).

56. H. Koo, S. Yoon, O.-J. Kwon, K.-E. Ko, D. Shin, S.-H. Bae, S.-H. Chang, and C. Park: Effect of lattice misfit on the transition temperature of $\mathrm{VO}_{2}$ thin film. J. Mater. Sci. 47, 6397-6401 (2012).

57. T.-H. Yang, R. Aggarwal, A. Gupta, H. Zhou, R.J. Narayan, and $J$. Narayan: Semiconductor-metal transition characteristics of $\mathrm{VO}_{2}$ thin films grown on C- and r-sapphire substrates. J. Appl. Phys. 107, 053514 (2010).

58. G. Garry, 0. Durand, and A. Lordereau: Structural, electrical and optical properties of pulsed laser deposited V02 thin films on R- and C-sapphire planes. Thin Solid Films 453-454, 427-430 (2004).

59. Y. Chang, J. Yang, Y. Kim, D. Kim, T. Noh, D.-W. Kim, E. Oh, B. Kahng, and J.-S. Chung: Surface versus bulk characterizations of electronic inhomogeneity in a $\mathrm{VO}_{2}$ thin film. Phys. Rev. B 76, 075118 (2007).

60. C.H. Griffiths: Influence of stoichiometry on the metal-semiconductor transition in vanadium dioxide. J. Appl. Phys. 45, 2201 (1974).

61. H.K. Kim, H. You, R.P. Chiarello, H.L.M. Chang, T.J. Zhang, and D.J. Lam: Finite-size effect on the first-order metal-insulator transition in $\mathrm{VO}_{2}$ films grown by metal-organic chemical-vapor deposition. Phys. Rev. B 47, 12900-12907 (1993). 
62. J. Jian, A. Chen, W. Zhang, and H. Wang: Sharp semiconductor-to-metal transition of $\mathrm{VO}_{2}$ thin films on glass substrates. J. Appl. Phys. 114, 244301 (2013).

63. S. Lee, T.L. Meyer, S. Park, T. Egami, and H.N. Lee: Growth control of the oxidation state in vanadium oxide thin films. Appl. Phys. Lett. 223515, 3-6 (2014)

64. H.-T. Zhang, L. Zhang, D. Mukherjee, Y.-X. Zheng, R.C. Haislmaier, N. Alem, and R. Engel-Herbert: Wafer-scale growth of $\mathrm{VO}_{2}$ thin films using a combinatorial approach. Nat. Commun. 6, 8475 (2015).

65. H-T. Zhang, C. Eaton, H. Ye, and R. Engel-Herbert: Phase stabilization of $\mathrm{VO}_{2}$ thin films in high vacuum using a co-deposition approach. J. Appl. Phys. 118, 185306 (2015).

66. Y. Cui and S. Ramanathan: Substrate effects on metal-insulator transition characteristics of rf-sputtered epitaxial $\mathrm{VO}_{2}$ thin films. J. Vac. Sci. Technol. A, Vac. Surf. Film 29, 041502 (2011).

67. Y. Shigesato, M. Enomoto, and H. Odaka: Thermochromic $\mathrm{VO}_{2}$ films deposited by RF magnetron sputtering using $\mathrm{V}_{2} \mathrm{O}_{3}$ or $\mathrm{V}_{2} \mathrm{O}_{5}$ targets. Jpn. J. Appl. Phys. 39, 6016 (2000)

68. C. Ko, Z. Yang, and S. Ramanathan: Work function of vanadium dioxide thin films across the metal-insulator transition and the role of surface nonstoichiometry. ACS Appl. Mater. Interfaces 3, 3396-3401 (2011).

69. Y. Zhou and S. Ramanathan: Heteroepitaxial $\mathrm{VO}_{2}$ thin films on $\mathrm{GaN}$ : Structure and metal-insulator transition characteristics. J. Appl. Phys. 112, 074114 (2012)

70. Y. Zhao, J. Hwan Lee, Y. Zhu, M. Nazari, C. Chen, H. Wang, A. Bernussi, M. Holtz, and Z. Fan: Structural, electrical, and terahertz transmission properties of $\mathrm{VO}_{2}$ thin films grown on c-, r-, and m-plane sapphire substrates. J. Appl. Phys. 111, 053533 (2012).

71. A. Zimmers, L. Aigouy, M. Mortier, A. Sharoni, S. Wang, K. West, J. Ramirez, and I. Schuller: Role of thermal heating on the voltage induced insulator-metal transition in $\mathrm{VO}_{2}$. Phys. Rev. Lett. 110, 056601 (2013)

72. N-W. Zhu, M. Hu, X-X. Xia, X-Y. Wei, and J-R. Liang: Preparation and modification of $\mathrm{VO}_{2}$ thin film on R-sapphire substrate by rapid thermal process. Chin. Phys. B 23, 048108 (2014).

73. W. Zhang, K. Wang, L. Fan, L. Liu, P. Guo, C. Zou, J. Wang, H. Qian, K. Ibrahim, W. Yan, F. Xu, and Z. Wu: Hole carriers doping effect on the metal-insulator transition of $\mathrm{N}$-incorporated vanadium dioxide thin films. J. Phys. Chem. C 118, 12837-12844 (2014).

74. P.J. Hood and J.F. DeNatale: Millimeter-wave dielectric properties of epitaxial vanadium dioxide thin films. J. Appl. Phys. 70, 376 (1991).

75. K. West, J. Lu, and J. Yu: Growth and characterization of vanadium dioxide thin films prepared by reactive-biased target ion beam deposition. J. Vac. Sci. Technol. A 26, 133 (2008).

76. T. Maruyama and Y. Ikuta: Vanadium dioxide thin films prepared by chemical vapour deposition from vanadium(III) acetylacetonate. J. Mater. Sci. 28, 5073-5078 (1993).

77. Y.D. Ji, T.S. Pan, Z. Bi, W.Z. Liang, Y. Zhang, H.Z. Zeng, Q.Y. Wen, H.W. Zhang, C.L. Chen, Q.X. Jia, and Y. Lin: Epitaxial growth and metal-insulator transition of vanadium oxide thin films with controllable phases. Appl. Phys. Lett. 101, 071902 (2012).

78. P. Dagur, A.U. Mane, and S.A. Shivashankar: Thin films of $\mathrm{VO}_{2}$ on glass by atomic layer deposition: microstructure and electrical properties. J. Cryst. Growth 275, e1223-e1228 (2005)

79. G. Rampelberg, M. Schaekers, K. Martens, Q. Xie, D. Deduytsche, B. De Schutter, N. Blasco, J. Kittl, and C. Detavernier: Semiconductor-metal transition in thin $\mathrm{VO}_{2}$ films grown by ozone based atomic layer deposition. Appl. Phys. Lett. 98, 1-4 (2011).

80. S. Savo, Y. Zhou, G. Castaldi, M. Moccia, V. Galdi, S. Ramanathan, and $Y$. Sato: Reconfigurable anisotropy and functional transformations with $\mathrm{VO}_{2}$-based metamaterial electric circuits. Phys. Rev. B 91, 134105 (2015).

81. A.M. Ionescu and H. Riel: Tunnel field-effect transistors as energyefficient electronic switches. Nature 479, 329-337 (2011).

82. A. Jain and M.A. Alam: Prospects of hysteresis-free abrupt switching (0 mV/decade) in Landau switches. IEEE Trans. Electron Devices $\mathbf{6 0}$, 4269-4276 (2013).

83. S.M. Sze and K.K. Ng: Physics of Semiconductor Devices (John Wiley \& Sons, Inc., Hoboken, NJ, 2006).
84. V.V. Zhirnov and R.K. Cavin: Nanoelectronics: negative capacitance to the rescue? Nat. Nanotechnol. 3, 77-78 (2008).

85. H. Lu and A. Seabaugh: Tunnel field-effect transistors: state-of-the-art. IEEE J. Electron Devices Soc. 2, 44-49 (2014).

86. S. Salahuddin and S. Datta: Use of negative capacitance to provide voltage amplification for low power nanoscale devices. Nano Lett. 8, 405-410 (2008).

87. A.I. Khan, K. Chatterjee, B. Wang, S. Drapcho, L. You, C. Serrao, S.R. Bakaul, R. Ramesh, and S. Salahuddin: Negative capacitance in a ferroelectric capacitor. Nat. Mater. 14, 182-186 (2014).

88. W. Gao, A. Khan, X. Marti, C. Nelson, C. Serrao, J. Ravichandran, R. Ramesh, and S. Salahuddin: Room-temperature negative capacitance in a ferroelectric-dielectric superlattice heterostructure. Nano Lett. 14 5814-5819 (2014).

89. A. Islam Khan, D. Bhowmik, P. Yu, S. Joo Kim, X. Pan, R. Ramesh, and S. Salahuddin: Experimental evidence of ferroelectric negative capacitance in nanoscale heterostructures. Appl. Phys. Lett. 99, 113501 (2011).

90. G. Catalan, D. Jiménez, and A. Gruverman: Ferroelectrics: negative capacitance detected. Nat. Mater. 14, 137-139 (2015).

91. R. Pandey, H. Madan, H. Liu, V. Chobpattana, M. Barth, B. Rajamohanan, M.J. Hollander, T. Clark, K. Wang, J.-H. Kim, D. Gundlach, K.P. Cheung, J. Suehle, R. Engel-Herbert, S. Stemmer, and S. Datta: Demonstration of p-type In0.7Ga0.3As/GaAs0.35Sb0.65; and n-type GaAs0.4Sb0.6/ In0.65Ga0.35As complimentary heterojunction vertical tunnel FETs for ultra-low power logic. In 2015 Symp. VLSI Technol, VLSI Technol. T206-T207, IEEE, Washington, DC, 2015. doi: 10.1109/VLSIT.2015. 7223676.

92. K. Gopalakrishnan, P.B. Griffin, and J.D. Plummer: Impact ionization MOS (I-MOS)—Part I: device and circuit simulations. IEEE Trans. Electron Devices 52, 69-76 (2005).

93. K. Gopalakrishnan, R. Woo, C. Jungemann, P.B. Griffin, and J.D. Plummer: Impactionization MOS (I-MOS)-Part II: Experimental results. IEEE Trans. Electron Devices 52, 77-84 (2005).

94. T.N. Theis, P.M. Solomon, R.H. Dennard, F.H. Gaensslen, V.L. Rideout, E. Bassous, A.R. LeBlanc, W. Haensch, S. Banerjee, W. Richardson, J. Coleman, A. Chatterjee, J. Appenzeller, Y.-M. Lin, J. Knoch, P. Avouris, S. Salahuddin, S. Datta, J. Li, T. Kopp, J. Mannhart, J. Mannhart, and D.G. Schlom: It's time to reinvent the transistor! Science 327, 1600-1601 (2010).

95. I.H. Inoue and M.J. Rozenberg: Taming the Mott transition for a novel Mott transistor. Adv. Funct. Mater. 18, 2289-2292 (2008).

96. H.Y. Hwang, Y. Iwasa, M. Kawasaki, B. Keimer, N. Nagaosa, and Y. Tokura: Emergent phenomena at oxide interfaces. Nat. Mater. 11, 103-113 (2012).

97. D.M. Newns, J.A. Misewich, C.C. Tsuei, A. Gupta, B.A. Scott, and A. Schrott: Mott transition field effect transistor. Appl. Phys. Lett. 73, 780 (1998).

98. D. Ruzmetov, G. Gopalakrishnan, C. Ko, V. Narayanamurti, and S. Ramanathan: Three-terminal field effect devices utilizing thin film vanadium oxide as the channel layer. J. Appl. Phys. 107, 114516 (2010).

99. S. Hormoz and S. Ramanathan: Limits on vanadium oxide Mott metalinsulator transition field-effect transistors. Solid State Electron. 54, 654659 (2010).

100.H.-T. Kim, B.-G. Chae, D.-H. Youn, S.-L. Maeng, G. Kim, K.-Y. Kang, and Y.-S. Lim: Mechanism and observation of Mott transition in $\mathrm{VO}_{2}$-based two- and three-terminal devices. New J. Phys. 6, 52-52 (2004).

101.K. Martens, J.W. Jeong, N. Aetukuri, C. Rettner, N. Shukla, E. Freeman, D.N. Esfahani, F.M. Peeters, T. Topuria, P.M. Rice, A. Volodin, B. Douhard, W. Vandervorst, M.G. Samant, S. Datta, and S.S.P. Parkin: Field effect and strongly localized carriers in the metal-insulator transition material V0 2 . Phys. Rev. Lett. 115, 196401 (2015).

102.Y. Zhou and S. Ramanathan: Relaxation dynamics of ionic liquid- $-\mathrm{VO}_{2}$ interfaces and influence in electric double-layer transistors. J. Appl. Phys. 111, 084508 (2012).

103.N. Shukla, A.V. Thathachary, A. Agrawal, H. Paik, A. Aziz, D.G. Schlom, S.K. Gupta, R. Engel-Herbert, and S. Datta: A steep-slope transistor based on abrupt electronic phase transition. Nat. Commun. 6, 7812 (2015). 
104.H. Wen, L. Guo, E. Barnes, J.H. Lee, D.A. Walko, R.D. Schaller, J.A. Moyer, R. Misra, Y. Li, E.M. Dufresne, D.G. Schlom, V. Gopalan, and J.W. Freeland: Structural and electronic recovery pathways of a photoexcited ultrathin $\mathrm{VO}_{2}$ film. Phys. Rev. B 88, 165424 (2013).

105. Ö. Türel, J.H. Lee, X. Ma, and K.K. Likharev: Neuromorphic architectures for nanoelectronic circuits. Int. J. Circuit Theory Appl. 32, 277-302 (2004).

106.C.W. Wu: Graph coloring via synchronization of coupled oscillators. IEEE Trans. Circuits Syst. I, Fundam. Theory Appl. 45, 974-978 (1998)

107.E.L. Lawler, J.K. Lenstra, A.H.G. Rinnooy Kan, and D.B. Shmoys: The Traveling Salesman Problem: A Guided Tour of Combinatorial Optimization (John Wiley \& Sons, New York, 1985).

108. R.W. Hölzel and K. Krischer: Pattern recognition with simple oscillating circuits. New J. Phys. 13, 073031 (2011).

109. E. Vassilieva, G. Pinto, J.A. de Barros, and P. Suppes: Learning pattern recognition through quasi-synchronization of phase oscillators. IEEE Trans. Neural Netw. 22, 84-95 (2011).

110.N. Shukla, A. Parihar, M. Cotter, M. Barth, X. Li, N. Chandramoorthy, H. Paik, D.G. Schlom, V. Narayanan, A. Raychowdhury, and S. Datta: Pairwise coupled hybrid vanadium dioxide-MOSFET (HVFET) oscillators for non-Boolean associative computing. In 2014 IEEE Int. Electron Devices Meet. 28.7.1-28.7.4, IEEE, San Francisco, CA, 2014.

111.D.L. Wang and D. Terman: Locally excitatory globally inhibitory oscillator networks. IEEE Trans. Neural Netw. 6, 283-286 (1995).

112.A. Parihar, N. Shukla, S. Datta, and A. Raychowdhury: Exploiting synchronization properties of correlated electron devices in a non-Boolean computing fabric for template matching. IEEE J. Emerg. Sel. Top. Circuits Syst. 4, 450-459 (2014).

113.D.J. Watts and S.H. Strogatz: Collective dynamics of "small-world" networks. Nature 393, 440-442 (1998).

114.M.D. Pickett, G. Medeiros-Ribeiro, and R.S. Williams: A scalable neuristor built with Mott memristors. Nat. Mater. 12, 114-117 (2012).

115.F. Dumas-Bouchiat, C. Champeaux, A. Catherinot, A. Crunteanu, and P. Blondy: Rf-microwave switches based on reversible semiconductormetal transition of $\mathrm{VO}_{2}$ thin films synthesized by pulsed-laser deposition. Appl. Phys. Lett. 91, 223505 (2007).

116.S.D. Ha, Y. Zhou, C.J. Fisher, S. Ramanathan, and J.P. Treadway: Electrical switching dynamics and broadband microwave characteristics of $\mathrm{VO}_{2}$ radio frequency devices. J. Appl. Phys. 113, 184501 (2013).

117. B. Nechay, R. Howell, E. Stewart, J. Parke, R. Freitag, H. Cramer, M. King, S. Gupta, J. Hartman, P. Borodulin, M. Snook, I. Wathuthanthri, K. Renaldo, and H.G. Henry: Optimizing performance of super-lattice castellated field effect transistors. In IEEE Compound Semiconductor Integrated Circuit Symp., La Jolla, CA, 2014, pp. 1-5.

118. H. Madan, H.-T. Zhang, M. Jerry, D. Mukherjee, N. Alem, R. EngelHerbert, and S. Datta: 26.5 Terahertz electrically triggered RF switch on epitaxial $\mathrm{VO}_{2}$-on-Sapphire (VOS) wafer. In 2015 IEEE Int. Electron Devices Meeting 9.3.1-9.3.4, IEEE, Washington, DC, 2015.

119.R.S. Howell, E.J. Stewart, R. Freitag, J. Parke, B. Nechay, C. Harlan, M. King, S. Gupta, J. Hartman, P. Borodulin, M. Snook, I. Wathuthanthri, P. Ralston, K. ReNaldo, and H.G. Henry: Low loss, high performance 1-18 GHz SPDT based on the novel super-lattice castellated field effect transistor (SLCFET). In Semiconductor, 2014 IEEE Compound \& Integrated Circuit Symp. (CSICS), IEEE, 2014, La Jolla, CA, 2014.

120.H. Kamitsuna, Y. Yamane, M. Tokumitsu, H. Sugahara, and M. Muraguchi: Low-power InP-HEMT switch ICs integrating miniaturized $2 \times 2$ switches for $10-\mathrm{Gb} / \mathrm{s}$ systems. IEEE J. Solid-State Circuits 41, 452-460 (2006).

121.Q. Xiao, G. Samiotes, T. Galluccio, and B. Rizzi: A high performance DC-20 GHz SPDT switch in a low cost plastic QFN package. In Microwave Integrated Circuits Conf., EuMA, 2009.

122.N. El-Hinnawy, P. Borodulin, E.B. Jones, B.P. Wagner, M.R. King, J. John Mason, J. Bain, J.T.E.S. Paramesh, R.S. Howell, M.J. Lee, and R.M. Young: $12.5 \mathrm{THz} F \mathrm{Fo}$ GeTe inline phase-change switch technology for reconfigurable RF and switching applications. In 2014 IEEE Compound Semiconductor Integrated Circuit Symp. (CSICS), Rome, Italy, n.d.

123. R. Wolf, A. Joseph, A. Botula, and J. Slinkman: A Thin-film SOI 180nm CMOS RF switch. In IEEE Topical Meeting on Silicon Monolithic
Integrated Circuits in RF Systems, 2009 (SiRF '09), San Diego, CA, 2009.

124.P.D. Grant, M.W. Denhoff, and R.R. Mansour: A comparison between RF MEMS switches and semiconductor switches. In Proc. of the 2004 Int. Conf. on MEMS, NANO and Smart Systems (ICMENS'04), Banff, Alberta, Canada, 2004.

125.C.F. Campbell and D.C. Dumka: Wideband high power GaN on SiC SPDT switch MMICs. In 2010 IEEE MTT-S Int. Microwave Symp. Digest, Anaheim, CA, 2010

126.A. Mennai, A. Bessaudou, F. Cosset, C. Guines, P. Blondy, A. Crunteanu: Bistable RF switches using $\mathrm{Ge}_{2} \mathrm{Sb}_{2} \mathrm{Te}_{5}$ phase change material. In 2014 IEEE MTT-S Int. Microwave Symp. (IMS2014), IEEE, Tampa, FL, 2014.

127.K-H. Hellwege and A.M. Hellwege: Landolt-Bornstein: Numerical Data and Functional Relationships in Science and Technology, Group III (Springer-Verlag Berlin and Heidelberg GmbH \& Co. K., 12a, 1978).

128. A.M. Glazer: The classification of tilted octahedra in perovskites. Acta Crystallogr. Sect. B, Struct. Crystallogr. Cryst. Chem. 28, 3384-3392 (1972).

129.A.M. Glazer: Simple ways of determining perovskite structures. Acta Crystallogr. Sect. A 31, 756-762 (1975).

130.D.I. Woodward and I.M. Reaney: Electron diffraction of tilted perovskites. Acta Crystallogr. Sect. B Struct. Sci. 61, 387-399 (2005).

131.M. O'Keeffe and B.G. Hyde: Some structures topologically related to cubic perovskite (E21), $\mathrm{ReO}_{3}$ (D09) and $\mathrm{Cu}_{3} \mathrm{Au}$ (L12). Acta Crystallogr. B 33, 3802-3813 (1977).

132.J.M. Rondinelli, S.J. May, and J.W. Freeland: Control of octahedral connectivity in perovskite oxide heterostructures: an emerging route to multifunctional materials discovery. MRS Bull. 37, 261-270 (2012).

133.L. Zhang, Y. Zhou, L. Guo, W. Zhao, A. Barnes, H.-T. Zhang, C. Eaton, Y. Zheng, M. Brahlek, H.F. Haneef, N.J. Podraza, M.H.W. Chan, V. Gopalan, K.M. Rabe, and R. Engel-Herbert: Correlated metals as transparent conductors. Nat. Mater. 15, 204-215 (2016).

134.T. Yoshida, K. Tanaka, H. Yagi, A. Ino, H. Eisaki, A. Fujimori, and Z.X. Shen: Direct observation of the mass renormalization in $\mathrm{SrVO}_{3}$ by angle resolved photoemission spectroscopy. Phys. Rev. Lett. 95, 1-4 (2005).

135.T. Yoshida, M. Kobayashi, K. Yoshimatsu, H. Kumigashira, and A. Fujimori: Correlated electronic states of $\mathrm{SrVO}_{3}$ revealed by angle-resolved photoemission spectroscopy. J. Electron Spectrosc. Relat. Phenom. 208, 11-16 (2016).

136.A. Fujimori, I. Hase, H. Namatame, Y. Fujishima, Y. Tokura, K. Takegahara, and F.M.F. de Groot: Evolution of the spectral function in Mott-Hubbard systems with $\mathrm{d}^{1}$ configuration. Phys. Rev. Lett. 69, 1796-1799 (1992).

137.K. Morikawa, T. Mizokawa, K. Kobayashi, A. Fujimori, H. Eisaki, S. Uchida, F. Iga, and Y. Nishihara: Spectral weight transfer and mass renormalization in Mott-Hubbard systems $\mathrm{SrVO}_{3}$ and $\mathrm{CaVO}_{3}$ : influence of long-range Coulomb interaction. Phys. Rev. B 52, 13711-13714 (1995).

138.R.J.O. Mossanek, M. Abbate, T. Yoshida, A. Fujimori, Y. Yoshida, N. Shirakawa, H. Eisaki, S. Kohno, and F.C. Vicentin: Evolution of the spectral weight in the Mott-Hubbard series $\mathrm{SrVO}_{3}-\mathrm{CaVO}_{3}-\mathrm{LaVO}_{3}-\mathrm{YVO}_{3}$. Phys. Rev. B 78, 075103 (2008).

139.J.B. Goodenough and M. Longo: Crystallogr. Prop. Compd. with perovskite or perovskite-related Struct, edited by K.-H. Hellwege and A.M. Hellwege (SpringerMaterials-The Landolt-Börnstein Database, Berlin, 1970), p. 126.

140.T. Yoshida, M. Hashimoto, T. Takizawa, A. Fujimori, M. Kubota, K. Ono, and $H$. Eisaki: Mass renormalization in the bandwidth-controlled Mott-Hubbard systems $\mathrm{SrVO}_{3}$ and $\mathrm{CaVO}_{3}$ studied by angle-resolved photoemission spectroscopy. Phys. Rev. B, Condens. Matter Mater. Phys. 82, 3-7 (2010).

141.E. Pavarini, S. Biermann, A. Poteryaev, A.I. Lichtenstein, A. Georges, and 0.K. Andersen: Mott transition and suppression of orbital fluctuations in orthorhombic $3 d^{1}$ perovskites. Phys. Rev. Lett. 92, 176403-1-176403-4 (2004).

142.I.H. Inoue, I. Hase, Y. Aiura, A. Fujimori, Y. Haruyama, T. Maruyama, and Y. Nishihara: Systematic development of the spectral function in the $3 d^{1}$ 
Mott-Hubbard system $\mathrm{Ca}_{1-x} \mathrm{Sr}_{x} \mathrm{VO}_{3}$. Phys. Rev. Lett. 74, 2539-2542 (1995).

143. H. Makino: Specific heat of a single-crystalline $3 d^{1}$ perovskite. J. Phys. Condens. Matter. 10, 11541-11545 (1998).

144. R. Eguchi, T. Kiss, S. Tsuda, T. Shimojima, T. Mizokami, T. Yokoya, A. Chainani, S. Shin, I. H. Inoue, T. Togashi, S. Watanabe, C.Q. Zhang, C.T. Chen, M. Arita, K. Shimada, H. Namatame, and M. Taniguchi: Bulk- and surface-sensitive high-resolution photoemission study of two Mott-Hubbard systems: $\mathrm{SrVO}_{3}$ and $\mathrm{CaVO}_{3}$. Phys. Rev. Lett. 96, 076402 (2006)

145.W.C. Sheets, B. Mercey, and W. Prellier: Effect of charge modulation in $\left(\mathrm{LaVO}_{3}\right)_{\mathrm{m}}\left(\mathrm{SrVO}_{3}\right)_{\mathrm{n}}$ superlattices on the insulator-metal transition. Appl. Phys. Lett. 91, 192102 (2007).

146.M. Liberati, R.V. Chopdekar, V. Mehta, E. Arenholz, and Y. Suzuki: Epitaxial growth and characterization of $\mathrm{CaVO}_{3}$ thin films. J. Magn. Magn. Mater. 321, 2852-2854 (2009).

147.D.-W. Kim, D.H. Kim, T.W. Noh, K. Char, J.-H. Park, K.-B. Lee, and H.-D. Kim: Interface chemistry and electrical properties of $\mathrm{SrVO}_{3} /$ $\mathrm{LaAlO}_{3}$ heterostructures. J. Appl. Phys. 88, 7056 (2000).

148.D. Kim, D.-W. Kim, B. Kang, T. Noh, D. Lee, K.-B. Lee, and S. Lee: Electrical properties of $\mathrm{SrVO}_{3} / \mathrm{SrTiO}_{3}$ superlattices grown by laser molecular beam epitaxy. Solid State Commun. 114, 473-476 (2000).

149. Y. Hotta, T. Susaki, and H. Hwang: Polar discontinuity doping of the $\mathrm{LaVO}_{3} / \mathrm{SrTiO}_{3}$ interface. Phys. Rev. Lett. 99, 236805 (2007).

150.M. Takizawa, Y. Hotta, T. Susaki, Y. Ishida, H. Wadati, Y. Takata, K. Horiba, M. Matsunami, S. Shin, M. Yabashi, K. Tamasaku, Y. Nishino, T. Ishikawa, A. Fujimori, and H.Y. Hwang: Spectroscopic evidence for competing reconstructions in polar multilayers $\mathrm{LaAlO}_{3} / \mathrm{LaVO}_{3} / \mathrm{LaAlO}$. Phys. Rev. Lett. 102, 236401 (2009).

151.A. Masuno, T. Terashima, and M. Takano: Epitaxial growth of perovskitetype $\mathrm{LaVO}_{3}$ thin films on various substrates by the PLD method. Solid State Ion. 172, 275-278 (2004)

152.C. He, T.D. Sanders, M.T. Gray, F.J. Wong, V.V. Mehta, and Y. Suzuki: Metal-insulator transitions in epitaxial $\mathrm{LaVO}_{3}$ and $\mathrm{LaTiO}_{3}$ films. Phys. Rev. B 86, 081401 (2012).

153. H. Ishiwara and K. Jyokyu: Formation of conductive $\mathrm{SrVO}_{3}$ films on Si substrates. Jpn. J. Appl. Phys. 30, L2059-L2061 (1991).

154.M. Gu, S.A. Wolf, and J. Lu: Two-dimensional Mott insulators in $\mathrm{SrVO}_{3}$ ultrathin films. Adv. Mater. Interfaces 1, 1300126 (2014).

155.M. Gu, J. Laverock, B. Chen, K.E. Smith, S.A. Wolf, and J. Lu: Metal-insulator transition induced in $\mathrm{CaVO}_{3}$ thin films. J. Appl. Phys. 113, 133704 (2013)

156. H. Koinuma, M. Yoshimoto, H. Nagata, and T. Tsukahara: Fabrication and anomalous conducting behavior of atomically regulated superlattices. Solid State Commun. 80, 9-13 (1991).

157.J.Y. Tsao: Materials Fundamentals of Molecular Beam Epitaxy (Academic Press, San Diego, CA, 1993).

158. B. Jalan, R. Engel-Herbert, N.J. Wright, and S. Stemmer: Growth of highquality $\mathrm{SrTiO}_{3}$ films using a hybrid molecular beam epitaxy approach. J. Vac. Sci. Technol. A, Vac. Surf. Film 27, 461 (2009).

159.R. Engel-Herbert: Molecular Beam Epitaxy From Research to Mass Production, Chapter 17 (Elsevier Science, Waltham, MA and Oxford, UK, 2013).

160.J.A. Moyer, C. Eaton, and R. Engel-Herbert: Highly conductive $\mathrm{SrVO}_{3}$ as a bottom electrode for functional perovskite oxides. Adv. Mater. 25, 3578-3582 (2013).

161.M. Brahlek, L. Zhang, C. Eaton, H-T. Zhang, and R. Engel-Herbert: Accessing a growth window for $\mathrm{SrVO}_{3}$ thin films. Appl. Phys. Lett. 107, 143108 (2015)

162.C. Eaton, L. Zhang, M. Brahlek, J.M. Lapano, and R. Engel-Herbert: Self-regulated growth of $\mathrm{CaVO}_{3}$ by hybrid molecular beam epitaxy. (Under Rev.) J. Vac. Sci. Technol. A (2016).

163.H-T. Zhang, L.R. Dedon, L.W. Martin, and R. Engel-Herbert: Self-regulated growth of $\mathrm{LaVO}_{3}$ thin films by hybrid molecular beam epitaxy. Appl. Phys. Lett. 106, 233102 (2015).

164.H.-T. Zhang, M. Brahlek, X. Ji, S. Lei, J.M. Lapano, J.W. Freeland, V. Gopalan, and R. Engel-Herbert: High quality $\mathrm{LaVO}_{3}$ films for photovoltaic applications. ACS Appl. Mater. Interfaces (Under Rev).
165.M. Brahlek, L. Zhang, H.-T. Zhang, J. Lapano, L.R. Dedon, L.W. Martin, and R. Engel-Herbert: Mapping growth windows in quaternary perovskite oxide systems by hybrid molecular beam epitaxy. Appl. Phys. Lett. 109, 101903 (2016).

166.K. Yoshimatsu, K. Horiba, H. Kumigashira, T. Yoshida, A. Fujimori, and M. Oshima: Metallic quantum well states in artificial structures of strongly correlated oxide. Science 333, 319-322 (2011).

167.K. Yoshimatsu, T. Okabe, H. Kumigashira, S. Okamoto, S. Aizaki, A. Fujimori, and M. Oshima: Dimensional-crossover-driven metal-insulator transition in $\mathrm{SrVO}_{3}$ ultrathin films. Phys. Rev. Lett. 104, 1-4 (2010).

168.Z. Zhong, M. Wallerberger, J.M. Tomczak, C. Taranto, N. Parragh, A. Toschi, G. Sangiovanni, and K. Held: Electronics with correlated oxides: $\mathrm{SrVO}_{3} / \mathrm{SrTiO}_{3}$ as a Mott transistor. Phys. Rev. Lett. 114, 246401 (2015).

169.J. Son, P. Moetakef, B. Jalan, 0. Bierwagen, N. J. Wright, R. EngelHerbert, and S. Stemmer: Epitaxial $\mathrm{SrTiO}_{3}$ films with electron mobilities exceeding $30,000 \mathrm{~cm}^{2} \mathrm{~V}^{-1} \mathrm{~s}^{-1}$. Nat. Mater. 9, 482-484 (2010).

170.K. Yoshimatsu, T. Okabe, H. Kumigashira, S. Okamoto, S. Aizaki, A. Fujimori, and M. Oshima: Dimensional-crossover-driven metal-insulator transition in $\mathrm{SrVO}_{3}$ ultrathin films. Phys. Rev. Lett. 104, 147601 (2010).

171.K. Ellmer: Past achievements and future challenges in the development of optically transparent electrodes. Nat. Photonics 6, 808-816 (2012).

172.R.B.H. Tahar, T. Ban, Y. Ohya, and Y. Takahashi: Tin doped indium oxide thin films: electrical properties. J. Appl. Phys. 83, 2631-2645 (1998).

173.S. Miyasaka, Y. Okimoto, and Y. Tokura: Anisotropy of Mott-Hubbard gap transitions due to spin and orbital ordering in $\mathrm{LaVO}_{3}$ and $\mathrm{YVO}_{3}$. J. Phys. Soc. Japan 71, 2086-2089 (2002).

174.M. Sage, G. Blake, C. Marquina, and T. Palstra: Competing orbital ordering in $\mathrm{RVO}_{3}$ compounds: high-resolution $\mathrm{x}$-ray diffraction and thermal expansion. Phys. Rev. B 76, 195102 (2007).

175.J.B. Goodenough: Theory of the role of covalence in the perovskite-type manganites [La,M(II)] $\mathrm{MnO}_{3}$. Phys. Rev. 100, 564-573 (1955).

176.J. Kanamori: Superexchange interaction and symmetry properties of electron orbitals. J. Phys. Chem. Solids 10, 87-98 (1959).

177.E. Assmann, P. Blaha, R. Laskowski, K. Held, S. Okamoto, and G. Sangiovanni: Oxide heterostructures for efficient solar cells. Phys. Rev. Lett. 110, 78701 (2013).

178. L. Wang, Y. Li, A. Bera, C. Ma, F. Jin, K. Yuan, W. Yin, A. David, W. Chen, W. Wu, W. Prellier, S. Wei, and T. Wu: Device performance of the Mott insulator $\mathrm{LaVO}_{3}$ as a photovoltaic material. Phys. Rev. Appl. 3, 064015 (2015)

179.X. Mathew, G.W. Thompson, V.P. Singh, J.C. McClure, S. Velumani, N.R. Mathews, and P.J. Sebastian: Development of CdTe thin films on flexible substrates-A review. Sol. Energy Mater. Sol. Cells 76, 293303 (2003).

180.M.D. Scafetta, A.M. Cordi, J.M. Rondinelli, and S.J. May: Band structure and optical transitions in $\mathrm{LaFeO}_{3}$ : theory and experiment. J. Phys. Condens. Matter 52, 505502 (2014).

181.S. Kachi, K. Kosuge, and H. Okinaka: Metal-insulator transition in $\mathrm{V}_{\mathrm{n}} \mathrm{O}_{2 \mathrm{n}-1}$. J. Solid State Chem. 6, 258-270 (1973).

182.K. Kawashima, Y. Ueda, K. Kosuge, and S. Kachi: Crystal growth and some electric properties of $\mathrm{V}_{6} \mathrm{O}_{13}$. J. Cryst. Growth 26, 321-322 (1974).

183.H. Okinaka, K. Kosuge, S. Kachi, K. Nagasawa, Y. Bando, and T. Takada: Electrical properties of $\mathrm{V}_{8} \mathrm{O}_{15}$ single crystal. Phys. Lett. A 33, 370-371 (1970).

184.N. Shukla, A. Parihar, E. Freeman, H. Paik, G. Stone, V. Narayanan, H. Wen, Z. Cai, V. Gopalan, R. Engel-Herbert, D.G. Schlom, A. Raychowdhury, and S. Datta: Synchronized charge oscillations in correlated electron systems. Sci. Rep. 4, 4964 (2014). 